\title{
Whole grain, bran and cereal fibre consumption and CVD: a systematic review
}

\author{
Eden M. Barrett ${ }^{1,2 *}$, Marijka J. Batterham ${ }^{2,3}$, Sumantra Ray ${ }^{4}$ and Eleanor J. Beck ${ }^{1,2}$ \\ ${ }^{1}$ School of Medicine, University of Wollongong, Wollongong, NSW 2522, Australia \\ ${ }^{2}$ Illawarra Health and Medical Research Institute, University of Wollongong, Wollongong, NSW 2522, Australia \\ ${ }^{3}$ School of Mathematics and Applied Statistics, University of Wollongong, Wollongong, NSW 2522, Australia \\ ${ }^{4}$ NNEdPro Global Centre for Nutrition and Health (affiliated with: Cambridge University Health Partners, Wolfson College \\ Cambridge and the British Dietetic Association), St John's Innovation Centre, Cowley Road, Cambridge CB4 OWS, UK
}

(Submitted 11 September 2018 - Final revision received 10 December 2018 - Accepted 3 February 2019)

\section{Abstract}

Whole grain intake is associated with lower CVD risk in epidemiological studies. It is unclear to what extent cereal fibre, located primarily within the bran, is responsible. This review aimed to evaluate association between intake of whole grain, cereal fibre and bran and CVD risk. Academic databases were searched for human studies published before March 2018. Observational studies reporting whole grain and cereal fibre or bran intake in association with any CVD-related outcome were included. Studies were separated into those defining whole grain using a recognised definition (containing the bran, germ and endosperm in their natural proportions) (three studies, seven publications) and those using an alternative definition, such as including added bran as a whole grain source (eight additional studies, thirteen publications). Intake of whole grain, cereal fibre and bran were similarly associated with lower risk of CVD-related outcomes. Within the initial analysis, where studies used the recognised whole grain definition, results were less likely to show attenuation after adjustment for cereal fibre content. The fibre component of grain foods appears to play an important role in protective effects of whole grains. Adjusting for fibre content, associations remained, suggesting that additional components within the whole grain, and the bran component, may contribute to cardio-protective association. The limited studies and considerable discrepancy in defining and calculating whole grain intake limit conclusions. Future research should utilise a consistent definition and methodical approach of calculating whole grain intake to contribute to a greater body of consistent evidence surrounding whole grains.

Key words: Whole grains: Bran: Fibre: CVD: Systematic reviews

CVD is a major cause of death worldwide, accounting for $31 \%$ of deaths globally in $2015^{(1)}$. As well as non-preventable risk factors such as age and ethnicity, factors such as dyslipidaemia, hypertension, abdominal obesity and pre-existing type 2 diabetes mellitus (T2DM) can contribute to the risk of $\mathrm{CVD}^{(2,3)}$. Diet can play a role in managing and reducing the risk of these associated factors, and a diet high in whole grains is consistently found to be protective against $\mathrm{CVD}^{(4,5)}$. 'Whole grains' refer to grains that contain the relative proportions of bran, germ and endosperm as they would occur naturally in the intact grain; as opposed to refined grains that have the bran and germ removed during processing. The precise mechanisms behind the cardio-protective effects of whole grains are not yet completely understood; however, many of those proposed involve the role of the cereal fibre located primarily within the bran component. For example, whole grains high in soluble fibre, such as oats and barley, may reduce the risk of CVD by decreasing serum LDL-cholesterol and improving blood glucose and insulin responses ${ }^{(6)}$.
While it is certain that the cereal fibre plays a role in the cardio-protective association of whole grain foods, there is still debate as to what extent it is responsible. There are other constituents of the whole grain that may contribute to lower risk of CVD, including $\mathrm{Mg}^{(7)}$, vitamin $\mathrm{E}$ and bioactive compounds such as phyto-oestrogens ${ }^{(8)}$. It is commonly considered that the contribution of these additional constituents, or perhaps, a synergistic effect of consuming the grain's nutrients and bioactive compounds together, is greater than any benefits of cereal fibre alone, if it were consumed outside of the whole grain.

As the bran component of the grain, removed within the refining process, contains the overwhelming majority of cereal fibre as well as these other constituents within the aleurone layer, comparing whole grain with refined grain intake is not appropriate to address this question. Instead, research needs to compare or adjust whole grain intake with cereal fibre intake and bran specifically.

Abbreviations: CRP, C-reactive protein; HPFS, Health Professionals Follow-up Study; HR, hazard ratio; NHS, Nurses' Health Study; RR, relative risk; T2DM, type 2 diabetes mellitus.

* Corresponding author: E. M. Barrett, fax +61242214844, email emb952@uow.edu.au 
However, a major issue in attempts to investigate comparative association of cereal fibre, whole grain and bran to date, is the lack of consistency in definition and calculation of whole grains. Many of the large meta-analyses providing evidence for the health association of whole grains draw conclusions based on studies that define whole grains in various ways, including those that consider added bran and germ as whole grain sources $^{(9,10)}$. In addition, many studies determined whole grain intake based on categorisation of mixed foods as either a whole grain or a refined grain food rather than calculating whole grain content as a dry weight of the ingredients within all foods. This method has the potential to overestimate the contribution to whole grain intake from some foods categorically considered whole grain, for example, dark breads that may use some refined flours. Alternative whole grain food definitions such as breakfast cereals that contain at least $25 \%$ whole grain or bran $^{(11)}$, or the recently developed $30 \%$ cut-off recommended by the HEALTHGRAIN forum ${ }^{(12)}$, have value in food labelling but are problematic in defining precise levels of intake in research. These methods can fail to capture the whole grain consumed in small amounts from food which do not meet these definitions but which could potentially accumulate to a significant contribution to total intake.

Therefore, to investigate the true cardio-protective association of whole grains, we considered the extent and quality of evidence for whole grains analysed only when using a recognised definition (namely, containing the bran, germ and endosperm in their natural proportions) and calculated on a $\mathrm{g} / 100 \mathrm{~g}$ dry-weight basis. To provide insight into the comparative association of the cereal fibre component outside of the whole grain, we summarised evidence from studies that compared CVD-related health associations of intake of whole grain with intake of cereal fibre and/or bran.

Therefore, the aim of this review was to shed light on the relative contribution of the cereal fibre and bran components to the cardiovascular protective association found with whole grain intake.

\section{Methods}

The systematic literature review was completed in accordance with the Preferred Reporting Items for Systematic Reviews and Meta-Analyses guidelines ${ }^{(13)}$. The details of the review were recorded and registered with PROSPERO (International Prospective Register of Systematic Reviews, http://www.crd.york. ac.uk/PROSPERO), registration number CRD42017069226 before commencement.

\section{Inclusion and exclusion criteria}

Articles were required to have considered both whole grain intake and cereal fibre and/or bran intake in association with CVD-related health outcomes in humans. Since there were only limited articles identified as meeting this criterion, no restrictions were placed on the CVD-related health outcomes examined and included. These could include but were not limited to disease biomarkers, anthropometric measures, morbidity and mortality. Results were restricted to those published in English and contained within peer-reviewed journals, and there were no restrictions based on the time of publication. Editorials, reviews, meta-analyses and conference abstracts were excluded.

Exposure - initial analysis. Articles must have reported on total whole grain intake, and those examining intake of only a defined set of whole grain foods or dietary patterns including whole grains were excluded. The definition of whole grains used was in accordance with that of American Association of Cereal Chemists International (AACCI): 'the intact, ground, cracked or flaked caryopsis, whose principal anatomical components - the starchy endosperm, germ and bran - are present in the same relative proportions as they exist in the intact caryopsis $^{,(14)}$. This definition is similar to those used in Europe and Australasia but critically defines whole grains, not whole grain foods. Also, it means that articles that included added bran or germ as a source of whole grain were excluded from the initial analysis.

Exposure - expanded analysis. Initial scoping found a limited amount of studies meeting the described inclusion criteria. Consequently, a second review of the evidence was conducted, expanding the criteria to include studies with a broader definition of whole grains. This expanded analysis includes studies that may not have defined whole grains as per the AACCI definition but may have utilised whole grain food definitions. For example, several articles that utilised a categorisation system of whole grain and refined grain foods, which categorised added bran and germ as a whole grain food, were included in an expanded analysis. Similarly, articles that met all other selection criteria but did not clearly explain how whole grain intake was defined or categorised were included in this expanded analysis.

Comparison. To be considered for inclusion, studies must have made a comparison of whole grain intake with cereal fibre and/ or bran intake. This may have been completed through assessment of cereal fibre/bran intake separately, adjustment of whole grain intake for cereal fibre/bran or by matching whole grain and refined grain intake based on cereal fibre/bran content. Bran was included as an alternative comparison as it is the portion of the grain containing the majority of the cereal fibre.

Study design. Due to wide disparity and potential differences between acute exposure and long-term exposure, this paper reports only the results of observational studies. Intervention studies will be reported in a separate review. A summary of the participants, exposure, comparisons, outcomes and study design is presented in Table 1 .

\section{Search term and strategy}

Relevant human journal articles were identified in medical databases Scopus, Web of Science, CINAHL plus, CENTRAL in Cochrane Library and PubMed published before March 2018 
Table 1. PICOS criteria for inclusion and exclusion of studies in initial analysis*

\begin{tabular}{ll}
\hline Parameter & Description \\
\hline Population & $\begin{array}{c}\text { Males and female humans of any age, health status, } \\
\text { socio-economic status and geographical location } \\
\text { Whole grain intake, whole grain defined according to } \\
\text { Exposure }\end{array}$ \\
$\begin{array}{c}\text { Cereal fibre intake or bran intake } \\
\text { Comparison }\end{array}$ & $\begin{array}{c}\text { Association of exposure/comparison on any } \\
\text { Outcomes } \\
\text { CVD-related health outcome }\end{array}$ \\
Study design & Observational studies \\
\hline
\end{tabular}

PICOS, population, intervention, comparator, outcome, study design; $\mathrm{AACCI}$, American Association of Cereal Chemists International.

* PICOS criteria, population, intervention (exposure), comparator, outcomes, study design.

† Expanded to include studies not meeting this definition in expanded analysis.

using the following key words: 'cardiovascular', 'coronary', 'stroke', 'cholesterol', 'triglycerides', 'lipids', 'obes*', 'overweight', 'glucose' and 'diabetes' in combination with 'wholegrain*' or 'grain*', as well as 'cereal*' or 'bran' and 'fibre' or 'fiber'. The full search strategy (SCOPUS) has been presented in online Supplementary material.

The titles of the articles were screened for inclusion by one author. The abstracts of remaining articles were then reviewed. After exclusion based on abstract, the full text of each potentially eligible article was retrieved. Two authors (E. M. B. and E. J. B.) separately assessed the full text studies for inclusion in the review. The reference lists of included articles were hand searched for additional studies that met the criteria.

\section{Data extraction}

One author (E. M. B.) extracted the required information and summarised the data in table format, which was reviewed by a second author (E. J. B.). Studies meeting the accepted definition of whole grains (initial analysis) were summarised separately for expanded analysis. Within both groups, whole grain, cereal fibre and bran intake data were extracted in separate tables. Each summary table included first author's last name, publication year, study name, participants, country in which the study was performed, CVD-related outcomes examined, highest and lowest median intake, covariate adjustments, main results and statistical significance. When bran intake data were extracted, the table also included whether the bran was total bran (naturally occurring plus added) or added bran.

\section{Quality assessment}

The design of each included study was identified and recorded. The National Health and Medical Research Council (NHMRC) levels of evidence guidelines ${ }^{(15)}$ were used to assign a level of evidence to each study based on design. In addition, the National Institutes of Health (NIH) Quality Assessment Tool for Observational Cohort and Cross-Sectional Studies ${ }^{(16)}$ was used to guide critical appraisal of articles. The NIH quality assessment tool provides a checklist of fourteen criteria that can be applied to cohort and cross-sectional studies to evaluate internal validity. Studies were assessed on the basis of research objective, study population specification, participation rate, inclusion/exclusion criteria, sample size justification, time frame, exposure and outcome measurement, blinding, attrition and confounders. The NIH tool then includes a subjective quality rating (good, fair or poor) that the reviewer decides on based on their overall evaluation, rather than a direct numerical ranking.

\section{Method of analysis}

Due to the wide range of CVD-related outcomes assessed within limited studies, a meta-analysis was not possible. Instead, studies were grouped based on comparison measure (cereal fibre or bran intake) and where possible, on health outcome assessed, and data were examined and synthesised qualitatively. The separation of articles within the initial and expanded analysis allows an examination of how the quality of evidence differs when restricted to articles using the currently accepted definition of whole grains. The quality assessment process was also used to inform the synthesis of results, with studies of higher quality guiding the interpretation of findings and subsequent discussion.

\section{Results}

Our search strategy identified 1909 articles (Fig. 1). Duplicates were removed and titles were screened for relevance, with 671 articles selected for screening of abstract. After screening for both title and abstracts, ninety-six full-text citations remained, with a further seventy-three articles excluded because they did not analyse both whole grain and cereal fibre or bran intake. One article was excluded as it measured only non-CVD-related outcomes, and two articles were excluded because they defined cereal fibre as the fibre sourced from breakfast cereals only. A further twelve articles were excluded from the initial analysis because they did not use the AACCI definition of whole grain and one article excluded based on no clear description of how whole grain foods were defined (later included within the expanded analysis). Seven articles (based on three studies) remained in the initial analysis.

When the expanded criterion was applied to include the articles that did not use the AACCI definition of whole grain, thirteen previously excluded articles (eight additional studies) were included (Fig. 1). They were previously excluded as eleven of these articles included added bran and/or germ as a source of whole grain, one article did not include whole grain bread as a source of whole grain intake, and one article did not provide sufficient detail as to how whole grain intake was defined.

A summary of articles exploring the associations between whole grain, cereal fibre and bran intake within the initial analysis are presented in Tables 2, 3 and 4, respectively. Similarly, Tables 5, 6 and 7 present a summary of articles in the same format for when the criteria were expanded. 


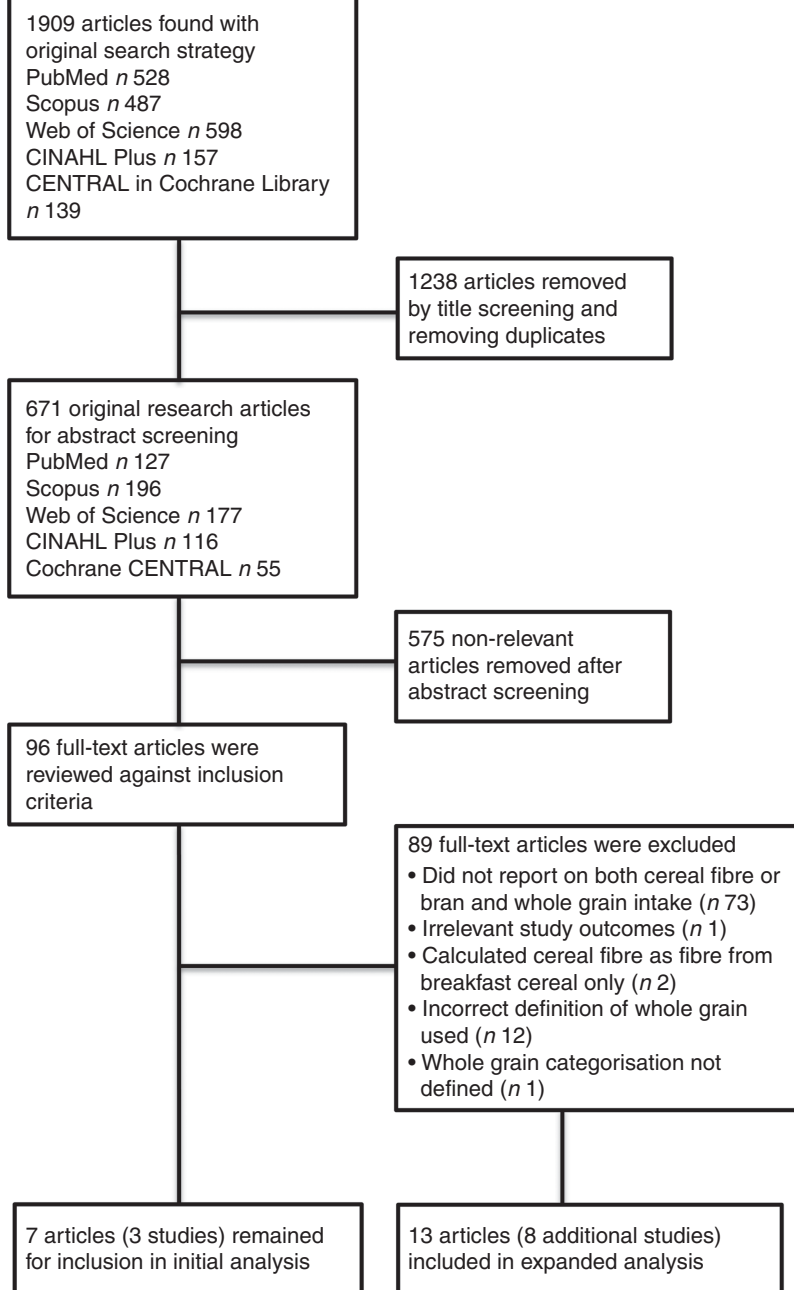

Fig. 1. Flow diagram of study selection process.

\section{Data extraction}

Within the initial analysis, seven articles, which were based on three primary studies, investigated various associations between whole grain, bran and cereal fibre intake and cardiovascularrelated outcomes. The primary studies were the Nurses' Health Study (NHS) I and II and the Health Professionals Follow-up Study (HPFS).

The following CVD-related outcomes were assessed in relation to whole grain and cereal fibre and/or bran intake within the initial analysis, each within single article: incidence of T2DM, CVD mortality and all-cause mortality in healthy participants and those with diabetes, hypertension, CHD, long-term weight gain and various inflammatory markers (Tables 2-4).

Within the expanded analysis (thirteen articles reporting on eight new studies), two articles directly compared outcomes between whole grain intake and bran intake and eight articles directly compared outcomes between whole grain intake and cereal fibre intake. One article adjusted whole grain intake for cereal fibre intake, and one article compared association between whole grain and non-whole grain intake matched on cereal fibre content. The expanded analysis investigated the incidence of T2DM (three articles), CVD mortality and all-cause mortality in healthy participants (two articles), incidence of CHD (one article), the metabolic syndrome (one article) and stroke (one article): and markers of CVD risk, anthropometric measures (three articles), blood lipid profile (two articles), blood glucose measures (three articles), inflammatory markers (one article) and change in coronary artery diameter and percentage stenosis (one article) (Tables 5-7).

\section{Quality assessment}

The level of evidence among the articles according to the NHMRC guidelines ranged from level II (twelve prospective cohort studies observing mortality or morbidity end points) to level IV (six cross sectional analysis studies) (Table 8). Five of the seven articles within the initial analysis were assigned a level II, while seven of the thirteen articles within the expanded analysis were assigned a level II.

While the majority of articles (10/19) were of good quality as determined using the NIH quality assessment tool, the ratio of good to fair quality was better in the initial analysis (6:1 v. 4:9 in the expanded analysis) (Table 8). The study design used often limited the quality that could be attained, with cross-sectional design unable to measure exposure before outcome and unable to assess associations over a sufficient time frame. In addition, articles that only assessed exposure once over the study period and articles that relied on self-reported outcome measures were often assessed as lower quality than those that assessed exposure multiple times and used validated measures.

\section{Initial analysis}

Of the seven articles that assessed whole grain intake in the initial analysis, six found a significant inverse association with CVD-related outcomes ${ }^{(5,17-21)}$, however, two of these found the association attenuated when adjusting for cereal fibre and whole grain constituents ${ }^{(5,20)}$. All six articles investigating bran intake found a significant inverse association with CVD-related outcomes $^{(4,5,17-21)}$, although one of these articles investigated two separate cohorts and found a non-significant inverse association in one cohort ${ }^{(19)}$. There were mixed results on the association with cereal fibre, with two articles showing a significant inverse association ${ }^{(17,21)}$ between intake and risk factors, and a third article showing no association ${ }^{(4)}$.

Whole grain intake compared with bran intake. The highest intake of whole grain was associated with $12 \%$ lower risk of CVD mortality (hazard ratio (HR) 0.88, 95\% CI 0.82, 0.96, $P=0.001$ ) and an $8 \%$ lower risk of all-cause mortality (HR 0.92 , $95 \%$ CI $0.89,0.96, P<0.001)$ compared with the lowest intake in a large combined cohort of the NHS I and the HPFS ${ }^{(18)}$. Within the same cohort, the association of high bran intake with lower risk of CVD mortality was slightly greater. A $20 \%$ lower risk for the highest intake of total bran (HR 0.80, 95\% CI 0.73, $0.87, P<0.001)$ and a $24 \%$ lower risk for the highest intake of added bran (HR 0.76, 95\% CI 0.70, 0.83, $P<0.001$ ) compared with lowest intake were observed. Similarly, high whole grain and total bran intake were both associated with lower risk of 


\section{N British Journal of Nutrition}

Table 2. Characteristics of studies within initial analysis exploring association of whole grain intake with outcomes related to CVD

\begin{tabular}{|c|c|c|c|c|c|c|c|c|c|c|}
\hline $\begin{array}{l}\text { Study first author, year } \\
\text { (reference) }\end{array}$ & Study & Cohort & Participants & Country & Outcome & $\begin{array}{l}\text { Highest } \\
\text { intake }\end{array}$ & $\begin{array}{l}\text { Lowest } \\
\text { intake }\end{array}$ & Covariates & Results & $P$ \\
\hline \multirow[t]{2}{*}{$\mathrm{He}, 2010^{(4)}$} & PCS & NHS I & $\begin{array}{l}7822 \mathrm{~F} \text { with } \\
\text { T2DM }\end{array}$ & USA & CVD death & $32.6 \mathrm{~g} / \mathrm{d}$ & $4.8 \mathrm{~g} / \mathrm{d}$ & $\begin{array}{l}\text { Age, smoking status, BMI, alcohol } \\
\text { intake, physical activity, parental } \\
\text { history of MI, menopausal status } \\
\text { and use of hormone therapy, } \\
\text { duration of diabetes mellitus. } \\
\text { Intake of total energy, PUFA, } \\
\text { saturated fat, trans fat, Mg and } \\
\text { folate }\end{array}$ & $\begin{array}{c}\text { RR } 0.70(95 \% \mathrm{Cl} \\
0.46,1.06)\end{array}$ & $P=0.07$ \\
\hline & & & & & All-cause death & & & & $\begin{array}{c}\text { RR } 0.89(95 \% \mathrm{Cl} \\
0.69,1.14)\end{array}$ & $P=0.11$ \\
\hline \multirow[t]{2}{*}{ Jensen, $2004^{(5)}$} & PCS & HPFS & $42850 \mathrm{M}$ & USA & $\mathrm{CHD}$ & $42.4 \mathrm{~g} / \mathrm{d}$ & $3.5 \mathrm{~g} / \mathrm{d}$ & $\begin{array}{l}\text { Age, BMI, added bran intake, added } \\
\text { germ intake, energy intake, } \\
\text { smoking status, alcohol intake, } \\
\text { physical activity, family history of } \\
\text { Ml, use of vitamin E supplement, } \\
\text { intake of PUFA, saturated fat, } \\
\text { trans fat, fruit, vegetables and fish }\end{array}$ & $\begin{array}{l}\text { HR } 0.84(95 \% \mathrm{Cl} \\
0.71,0.98)\end{array}$ & $P=0.02$ \\
\hline & & & & & & & & $\begin{array}{l}\text { Whole grain constituent intake: } \\
\text { dietary fibre, folate, glycaemic } \\
\text { load, dietary vitamin } \mathrm{B}_{6} \text {, vitamin } \mathrm{E} \text {, } \\
\mathrm{Mg} \text { and } \mathrm{Mn}\end{array}$ & $\begin{array}{c}\mathrm{HR} 0.85(95 \% \mathrm{Cl} \\
0.71,1.02)\end{array}$ & $P=0.06$ \\
\hline \multirow[t]{3}{*}{ Qi, 2006 ${ }^{(17)}$} & $\begin{array}{l}\text { Cross- } \\
\text { sectional }\end{array}$ & NHS I & $\begin{array}{l}902 \mathrm{~F} \text { with } \\
\text { T2DM }\end{array}$ & USA & CRP (mg/l) & $35.4 \mathrm{~g} / \mathrm{d}$ & $4.75 \mathrm{~g} / \mathrm{d}$ & $\begin{array}{l}\text { Age, BMI, smoking status, alcohol } \\
\text { intake, physical activity, aspirin } \\
\text { use, HbA1c, history of } \\
\text { hypertension or } \\
\text { hypercholesterolaemia, } \\
\text { postmenopausal hormone use, } \\
\text { glycaemic index and } \mathrm{Mg}\end{array}$ & $\begin{array}{l}\text { Q1 6.60 v. Q5 } \\
5.52\end{array}$ & $P=0.03$ \\
\hline & & & & & $\begin{array}{l}\text { ICAM-1 (ng/ml) } \\
\text { E-selectin (ng/ml) }\end{array}$ & & & & $\begin{array}{c}\text { Q1 } 316 v \text { v. Q5 } 306 \\
\text { Q1 } 65.6 \text { v. Q5 } \\
65.4\end{array}$ & $\begin{array}{l}P=0.19 \\
P=0.96\end{array}$ \\
\hline & & & & & TNF-R2 (pg/ml) & & & & $\begin{array}{l}\text { Q1 } 2647 \text { v. Q5 } \\
2435\end{array}$ & $P=0.017$ \\
\hline \multirow[t]{2}{*}{$\mathrm{Wu}, 2015^{(18)}$} & PCS & $\begin{array}{l}\text { NHS I and } \\
\text { HPFS }\end{array}$ & $\begin{array}{l}74341 \mathrm{~F} \\
\quad 43744 \mathrm{M}\end{array}$ & USA & CVD death & $47.8 \mathrm{~g} / \mathrm{d}$ & $4.2 \mathrm{~g} / \mathrm{d}$ & $\begin{array}{l}\text { Age, ethnicity, BMI, smoking status, } \\
\text { pack years smoked, years since } \\
\text { quitting for past smoker, alcohol } \\
\text { intake, physical activity, family } \\
\text { history of diabetes, cancer and } \\
\text { heart disease, multivitamin use, } \\
\text { aspirin use at least once per } \\
\text { week, history of hypertension, } \\
\text { high cholesterol or diabetes at } \\
\text { baseline, total energy intake, } \\
\text { postmenopausal status and } \\
\text { postmenopausal hormone use, } \\
\text { modified alternative healthy eating } \\
\text { index (excludes whole grains) }\end{array}$ & $\begin{array}{c}\text { HR } 0.88(95 \% \mathrm{Cl} \\
0.82,0.96)^{\star}\end{array}$ & $P=0.001$ \\
\hline & & & & & All-cause death & & & & $\begin{array}{c}\text { HR } 0.92(95 \% \mathrm{Cl} \\
0.89,0.96)^{\star}\end{array}$ & $P<0.001$ \\
\hline
\end{tabular}




\section{Nes British Journal of Nutrition}

Table 2. Continued

\begin{tabular}{|c|c|c|c|c|c|c|c|c|c|c|}
\hline $\begin{array}{l}\text { Study first author, year } \\
\text { (reference) }\end{array}$ & Study & Cohort & Participants & Country & Outcome & $\begin{array}{l}\text { Highest } \\
\text { intake }\end{array}$ & $\begin{array}{l}\text { Lowest } \\
\text { intake }\end{array}$ & Covariates & Results & $P$ \\
\hline \multirow[t]{2}{*}{ De Munter, $2007^{(19)}$} & PCS & NHS I & $167737 \mathrm{~F}$ & USA & T2DM & $31.2 \mathrm{~g} / \mathrm{d}$ & $3.7 \mathrm{~g} / \mathrm{d}$ & $\begin{array}{l}\text { Age, smoking status, physical } \\
\text { activity, alcohol intake, use of } \\
\text { hormone replacement therapy, } \\
\text { oral contraceptive use, family } \\
\text { history of T2DM, intake of coffee, } \\
\text { sugar-sweetened soft drinks and } \\
\text { fruit punch (non-alcoholic), total } \\
\text { energy intake, processed meat } \\
\text { intake, PUFA to saturated fat } \\
\text { intake ratio, BMl }\end{array}$ & $\begin{array}{l}\mathrm{RR} 0.75(95 \% \mathrm{Cl} \\
\quad 0.68,0.83)\end{array}$ & $P<0.001$ \\
\hline & & NHS II & & & & $39.9 \mathrm{~g} / \mathrm{d}$ & $6 \cdot 2 \mathrm{~g} / \mathrm{d}$ & & $\begin{array}{c}\text { RR } 0.86(95 \% \mathrm{Cl} \\
0.72,1.02)\end{array}$ & $P=0.03$ \\
\hline \multirow[t]{2}{*}{ Flint, $2009^{(20)}$} & PCS & HPFS & $31684 \mathrm{M}$ & USA & Hypertension & $46.0 \mathrm{~g} / \mathrm{d}$ & $3.3 \mathrm{~g} / \mathrm{d}$ & $\begin{array}{l}\text { Age, energy intake, family history of } \\
\text { CHD and hypertension, smoking } \\
\text { status, alcohol intake, marital } \\
\text { status, profession, height, fruit } \\
\text { and vegetable intake, Na intake, } \\
\text { physical activity, multivitamin use, } \\
\text { cholesterol screening }\end{array}$ & $\begin{array}{l}\text { RR } 0.81(95 \% \mathrm{Cl} \\
0.75,0.87)\end{array}$ & $P<0.0001$ \\
\hline & & & & & & & & Cereal fibre intake & $\begin{array}{c}\text { RR } 0.94(95 \% \mathrm{Cl} \\
0.84,1.05)\end{array}$ & $P=0.23$ \\
\hline Koh-Banerjee, $2004^{(21)}$ & PCS & HPFS & $27082 \mathrm{M}$ & USA & $\begin{array}{l}\text { Weight change over } 8 \\
\text { years }(\mathrm{kg})\end{array}$ & $\begin{array}{l}23 \cdot 8 \mathrm{~g} / \mathrm{d} \\
\text { change }\end{array}$ & $\begin{array}{r}-14 \cdot 2 \mathrm{~g} / \mathrm{d} \\
\text { change }\end{array}$ & $\begin{array}{l}\text { Age, baseline exposure, smoking } \\
\text { status, baseline weight, baseline } \\
\text { values and changes in refined } \\
\text { grains, total energy intake, } \\
\text { physical activity, alcohol intake, } \\
\text { protein intake and trans, } \\
\text { saturated, MUFA and PUFA } \\
\text { intake }\end{array}$ & $\begin{array}{r}\text { Q1 } 1.24(\mathrm{SD} 0.23) v . \\
\text { Q5 } 0.75(\mathrm{sD} 0.22)\end{array}$ & $P<0.0001$ \\
\hline
\end{tabular}

PCS, prospective cohort study; NHS, Nurses' Health Study; F, female; T2DM, type 2 diabetes mellitus; MI, myocardial infarction; RR, relative risk; HPFS, Health Professionals Follow-up Study; M, male; HR, hazard ratio; CRP, C-reactive protein; ICAM-1, intercellular adhesion molecule 1; TNF-R2, TNF receptor 2.

* Pooled HR for whole grain intake without added bran/germ. Located within Wu et al. ${ }^{(18)}$ online Supplementary Table S4. 
Table 3. Characteristics of studies within initial analysis exploring associations of cereal fibre intake with outcomes related to CVD

\begin{tabular}{|c|c|c|c|c|c|c|c|c|c|c|}
\hline $\begin{array}{l}\text { Study first author, } \\
\text { year (reference) }\end{array}$ & Study & Cohort & Participants & Country & Outcome & Highest intake & Lowest intake & Covariates & Results & $P$ \\
\hline \multirow[t]{2}{*}{$\mathrm{He}, 2010^{(4)}$} & PCS & NHS I & $7822 \mathrm{~F}$ with T2DM & USA & CVD death & $6.29 \mathrm{~g} / \mathrm{d}$ & $1.9 \mathrm{~g} / \mathrm{d}$ & $\begin{array}{l}\text { Age, smoking status, BMI, alcohol } \\
\text { intake, physical activity, parental } \\
\text { history of Ml, menopausal status } \\
\text { and use of hormone therapy, } \\
\text { duration of diabetes mellitus. } \\
\text { Intake of total energy, PUFA, } \\
\text { saturated fat, trans fat, Mg and } \\
\text { folate }\end{array}$ & $\begin{array}{c}\mathrm{RR} 0.89(95 \% \mathrm{Cl} \\
0.57,1.41)\end{array}$ & $P=0.41$ \\
\hline & & & & & All-cause death & & & & $\begin{array}{c}\mathrm{RR} 0.86(95 \% \mathrm{Cl} \\
0.66,1 \cdot 12)\end{array}$ & $P=0.10$ \\
\hline Qi, 2006 ${ }^{(17)}$ & Cross-sectional & NHS I & $902 \mathrm{~F}$ with T2DM & USA & CRP (mg/l) & N/A & $N / A$ & $\begin{array}{l}\text { Age, BMI, smoking status, alcohol } \\
\text { intake, physical activity, aspirin } \\
\text { use, HbA1c, history of } \\
\text { hypertension or } \\
\text { hypercholesterolaemia, } \\
\text { postmenopausal hormone use, } \\
\text { glycaemic index and Mg }\end{array}$ & N/A & $P=0.03$ \\
\hline $\begin{array}{l}\text { Koh-Banerjee, } \\
2004^{(21)}\end{array}$ & PCS & HPFS & $27082 \mathrm{M}$ & USA & $\begin{array}{l}\text { TNF-R2 }(\mathrm{pg} / \mathrm{ml}) \\
\text { Weight change } \\
\text { over } 8 \text { years } \\
(\mathrm{kg})\end{array}$ & $5.1 \mathrm{~g} / \mathrm{d}$ change & $-2.2 \mathrm{~g} / \mathrm{d}$ change & $\begin{array}{l}\text { Age, baseline exposure, smoking } \\
\text { status, baseline weight, baseline } \\
\text { values and changes in refined } \\
\text { grains, total energy intake, } \\
\text { physical activity, alcohol intake, } \\
\text { protein intake and trans, } \\
\text { saturated, MUFA and PUFA } \\
\text { intake }\end{array}$ & $\begin{array}{r}\text { Q1 } 1.10(\mathrm{sD} 0.20) v . \\
\text { Q5 0.76 (sD 0.20) }\end{array}$ & $\begin{array}{l}P=0.01 \\
P=0.0002\end{array}$ \\
\hline
\end{tabular}

PCS, prospective cohort study; NHS, Nurses' Health Study; F, female; T2DM, type 2 diabetes mellitus; MI, myocardial infarction; RR, relative risk; CRP, C-reactive protein; N/A, not available; TNF-R2, TNF receptor 2; HPFS, Health Professionals Follow-up Study; M, male. 


\section{NS British Journal of Nutrition}

Table 4. Characteristics of studies within initial analysis exploring associations of bran intake with outcomes related to CVD

\begin{tabular}{|c|c|c|c|c|c|c|c|c|c|c|c|}
\hline $\begin{array}{l}\text { Study first author, year } \\
\text { (reference) }\end{array}$ & Study & Cohort & Participants & Country & Outcome & Bran & $\begin{array}{l}\text { Highest } \\
\text { intake }\end{array}$ & $\begin{array}{l}\text { Lowest } \\
\text { intake }\end{array}$ & Covariates & Results & $P$ \\
\hline \multirow[t]{4}{*}{$\mathrm{He}, 2010^{(4)}$} & PCS & NHS I & $\begin{array}{l}7822 \mathrm{~F} \text { with } \\
\text { T2DM }\end{array}$ & USA & CVD death & Total $^{*}$ & $9.7 \mathrm{~g} / \mathrm{d}$ & $0.8 \mathrm{~g} / \mathrm{d}$ & $\begin{array}{l}\text { Age, smoking status, BMI, alcohol } \\
\text { intake, physical activity, parental } \\
\text { history of MI, menopausal status } \\
\text { and use of hormone therapy, } \\
\text { duration of diabetes mellitus. } \\
\text { Intake of total energy, PUFA, } \\
\text { saturated fat, trans fat, Mg and } \\
\text { folate }\end{array}$ & $\begin{array}{l}\text { RR } 0.65(95 \% \mathrm{Cl} \\
0.43,0.99)\end{array}$ & $P=0.04$ \\
\hline & & & & & & Added $\dagger$ & $\mathrm{N} / \mathrm{A}$ & $0.0 \mathrm{~g} / \mathrm{d}$ & & $\begin{array}{c}\text { RR } 0.36(95 \% \mathrm{Cl} \\
0.24,0.54)\end{array}$ & $P<0.001$ \\
\hline & & & & & All-cause death & Total & $9.7 \mathrm{~g} / \mathrm{d}$ & $0.8 \mathrm{~g} / \mathrm{d}$ & & $\begin{array}{l}\text { RR } 0.72(95 \% \mathrm{Cl} \\
0.56,0.92)\end{array}$ & $P=0.01$ \\
\hline & & & & & & Added & $\mathrm{N} / \mathrm{A}$ & $0.0 \mathrm{~g} / \mathrm{d}$ & & $\begin{array}{c}\text { RR } 0.45(95 \% \mathrm{Cl} \\
0.36,0.57)\end{array}$ & $P<0.001$ \\
\hline \multirow[t]{2}{*}{ Jensen, $2004^{(5)}$} & PCS & HPFS & $42850 \mathrm{M}$ & USA & $\mathrm{CHD}$ & Added & $6.85 \mathrm{~g} / \mathrm{d}$ & $0.0 \mathrm{~g} / \mathrm{d}$ & $\begin{array}{l}\text { Age, BMI, added germ intake, } \\
\text { energy intake, smoking status, } \\
\text { alcohol intake, physical activity, } \\
\text { family history of MI, use of vitamin } \\
\text { E supplement, intake of PUFA, } \\
\text { saturated fat, trans fat, fruit, } \\
\text { vegetables and fish }\end{array}$ & $\begin{array}{l}\text { HR } 0.72(95 \% \mathrm{Cl} \\
\quad 0.61,0.84)\end{array}$ & $P<0.001$ \\
\hline & & & & & & & & & $\begin{array}{l}\text { Whole grain constituent intake: } \\
\text { dietary fibre, folate, glycaemic } \\
\text { load, dietary vitamin } \mathrm{B}_{6} \text {, vitamin } \mathrm{E} \text {, } \\
\mathrm{Mg} \text { and } \mathrm{Mn}\end{array}$ & $\begin{array}{l}\text { HR } 0.71(95 \% \mathrm{Cl} \\
\quad 0.59,0.85)\end{array}$ & $P<0.001$ \\
\hline \multirow[t]{3}{*}{ Qi, 2006 ${ }^{(17)}$} & $\begin{array}{l}\text { Cross- } \\
\text { sectional }\end{array}$ & NHS I & $\begin{array}{l}902 \mathrm{~F} \text { with } \\
\text { T2DM }\end{array}$ & USA & CRP (mg/l) & Total & $10 \cdot 9 \mathrm{~g} / \mathrm{d}$ & $1.6 \mathrm{~g} / \mathrm{d}$ & $\begin{array}{l}\text { Age, BMI, smoking status, alcohol } \\
\text { intake, physical activity, aspirin } \\
\text { use, HbA1c, history of } \\
\text { hypertension or } \\
\text { hypercholesterolaemia, } \\
\text { postmenopausal hormone use, } \\
\text { glycaemic index and Mg }\end{array}$ & $\begin{array}{c}\text { Q1 } 6.29 v . \text { Q5 } \\
4.96\end{array}$ & $P=0.007$ \\
\hline & & & & & $\begin{array}{l}\text { ICAM-1 (ng/ml) } \\
\text { E-selectin (ng/ml) }\end{array}$ & & & & & $\begin{array}{c}\text { Q1 } 319 \text { v. Q5 } 303 \\
\text { Q1 67.2 v. Q5 } \\
68.1\end{array}$ & $\begin{array}{l}P=0.11 \\
P=0.86\end{array}$ \\
\hline & & & & & TNF-R2 (ng/ml) & & & & & $\begin{array}{l}\text { Q1 } 2603 \text { v. Q5 } \\
2462\end{array}$ & $P=0.06$ \\
\hline
\end{tabular}




\section{N British Journal of Nutrition}

Table 4. Continued

\begin{tabular}{|c|c|c|c|c|c|c|c|c|c|c|c|}
\hline $\begin{array}{l}\text { Study first author, year } \\
\text { (reference) }\end{array}$ & Study & Cohort & Participants & Country & Outcome & Bran & $\begin{array}{l}\text { Highest } \\
\text { intake }\end{array}$ & $\begin{array}{l}\text { Lowest } \\
\text { intake }\end{array}$ & Covariates & Results & $P$ \\
\hline \multirow[t]{4}{*}{$\mathrm{Wu}, 2015^{(18)}$} & PCS & $\begin{array}{l}\text { NHS I and } \\
\text { HPFS }\end{array}$ & $\begin{array}{l}74341 \mathrm{~F} \\
43744 \mathrm{M}\end{array}$ & USA & CVD death & Total & $\mathrm{N} / \mathrm{A}$ & $\mathrm{N} / \mathrm{A}$ & $\begin{array}{l}\text { Age, ethnicity, BMl, smoking status, } \\
\text { pack years smoked, years since } \\
\text { quitting for past smoker, alcohol } \\
\text { intake, physical activity, family } \\
\text { history of diabetes, cancer and } \\
\text { heart disease, multivitamin use, } \\
\text { aspirin use at least once per week, } \\
\text { history of hypertension, high } \\
\text { cholesterol or diabetes at baseline, } \\
\text { total energy intake, postmenopausal } \\
\text { status and postmenopausal } \\
\text { hormone use (for women), modified } \\
\text { alternative healthy eating index } \\
\text { (excludes whole grains) }\end{array}$ & $\begin{array}{l}\mathrm{HR} 0.80(95 \% \mathrm{Cl} \\
0.73,0.87)\end{array}$ & $P<0.001$ \\
\hline & & & & & & Added & $\geq 6.0 \mathrm{~g} / \mathrm{d}$ & $0 \mathrm{~g} / \mathrm{d}$ & & $\begin{array}{c}\text { HR } 0.76(95 \% \mathrm{Cl} \\
0.70,0.83) \ddagger\end{array}$ & $P<0.001$ \\
\hline & & & & & All-cause death & Total & $\mathrm{N} / \mathrm{A}$ & $\mathrm{N} / \mathrm{A}$ & & $\begin{array}{c}\text { HR } 0.94(95 \% \mathrm{Cl} \\
0.90,0.99) \S\end{array}$ & $P=0.02$ \\
\hline & & & & & & Added & $\geq 6.0 \mathrm{~g} / \mathrm{d}$ & $0 \mathrm{~g} / \mathrm{d}$ & & $\begin{array}{c}\text { HR } 0.93(95 \% \mathrm{Cl} \\
0.89,0.97) \ddagger\end{array}$ & $P=0.006$ \\
\hline \multirow[t]{2}{*}{ De Munter, $2007^{(19)}$} & PCS & NHS I & $167737 \mathrm{~F}$ & USA & T2DM & Total & $9.6 \mathrm{~g} / \mathrm{d}$ & $0.6 \mathrm{~g} / \mathrm{d}$ & $\begin{array}{l}\text { Age, smoking status, physical activity, } \\
\text { alcohol intake, use of hormone } \\
\text { replacement therapy, oral } \\
\text { contraceptive use, family history of } \\
\text { T2DM, intake of coffee, sugar- } \\
\text { sweetened soft drinks and fruit } \\
\text { punch (non-alcoholic), total energy } \\
\text { intake, processed meat intake, } \\
\text { PUFA:saturated fat intake ratio, BMI }\end{array}$ & $\begin{array}{c}\text { RR } 0.72(95 \% \mathrm{Cl} \\
0.65,0.80)\end{array}$ & $P<0.001$ \\
\hline & & NHS II & & & & Total & $12.0 \mathrm{~g} / \mathrm{d}$ & $1.1 \mathrm{~g} / \mathrm{d}$ & & $\begin{array}{c}\text { RR } 0.84(95 \% \mathrm{Cl} \\
0.71,1.00)\end{array}$ & $P=0.07$ \\
\hline Flint, $2009^{(20)}$ & PCS & HPFS & $31684 \mathrm{M}$ & USA & Hypertension & Total & $12.0 \mathrm{~g} / \mathrm{d}$ & $0.3 \mathrm{~g} / \mathrm{d}$ & $\begin{array}{l}\text { Age, energy intake, family history of } \\
\text { CHD and hypertension, smoking } \\
\text { status, alcohol intake, marital } \\
\text { status, profession, height, fruit } \\
\text { and vegetable intake, Na intake, } \\
\text { physical activity, multivitamin use, } \\
\text { cholesterol screening }\end{array}$ & $\begin{array}{l}\text { RR } 0.85(95 \% \mathrm{Cl} \\
0.78,0.92)\end{array}$ & $P=0.002$ \\
\hline Koh-Banerjee, $2004^{(21)}$ & PCS & HPFS & $27082 \mathrm{M}$ & USA & $\begin{array}{l}\text { 8-year weight } \\
\text { change }(\mathrm{kg})\end{array}$ & Added & $\begin{array}{l}7.3 \mathrm{~g} / \mathrm{d} \\
\text { change }\end{array}$ & $0.0 \mathrm{~g} / \mathrm{d}$ & $\begin{array}{l}\text { Age, baseline exposure, smoking } \\
\text { status, baseline weight, baseline } \\
\text { values and changes in refined } \\
\text { grains, total energy intake, } \\
\text { physical activity, alcohol intake, } \\
\text { protein intake and trans, } \\
\text { saturated, MUFA and PUFA } \\
\text { intake, baseline and changes in } \\
\text { natural bran }\end{array}$ & $\begin{array}{r}\text { Q1 } 1.16(\mathrm{sD} 0.23) v . \\
\text { Q3 1.00 (sD 0.20) }\end{array}$ & $P=0.01$ \\
\hline
\end{tabular}




\section{N British Journal of Nutrition}

Table 5. Characteristics of studies within expanded analysis exploring associations of whole grain intake with outcomes related to CVD

\begin{tabular}{|c|c|c|c|c|c|c|c|c|c|c|}
\hline $\begin{array}{l}\text { Study first author, } \\
\text { year (reference) }\end{array}$ & Study & Cohort & Participants & Country & Outcome & $\begin{array}{l}\text { Highest } \\
\text { intake }\end{array}$ & $\begin{array}{l}\text { Lowest } \\
\text { intake }\end{array}$ & Covariates & Results & $P$ \\
\hline \multirow[t]{11}{*}{ Jensen, 2006(22) } & \multirow[t]{11}{*}{ Cross-sectional } & \multirow[t]{11}{*}{ HPFS and NHS II } & \multirow[t]{11}{*}{$468 \mathrm{M}+470 \mathrm{~F}$} & \multirow[t]{11}{*}{ USA } & $\begin{array}{l}\text { Total cholesterol } \\
(\mathrm{mmol} / \mathrm{l})\end{array}$ & \multirow[t]{11}{*}{$43.8 \mathrm{~g} / \mathrm{d}$} & \multirow[t]{11}{*}{$8.2 \mathrm{~g} / \mathrm{d}$} & \multirow[t]{11}{*}{$\begin{array}{l}\text { Age, sex, total energy intake, } \\
\text { alcohol intake, smoking } \\
\text { status, BMI, physical activity, } \\
\text { hypercholesterolaemia, } \\
\text { intake of fruit, vegetable, } \\
\text { saturated fat, MUFA and } \\
\text { PUFA }\end{array}$} & $\begin{array}{r}\text { Q1 } 5.7 \text { (SE 0.08) } v \text {. } \\
\text { Q5 5.6 (SE 0.07) }\end{array}$ & $P=0.02$ \\
\hline & & & & & HDL-cholesterol & & & & Q1 1.7 (SE 0.03) V. & $P=0.05$ \\
\hline & & & & & $\begin{array}{l}\text { LDL-cholesterol } \\
(\mathrm{mmol} / \mathrm{l})\end{array}$ & & & & $\begin{array}{r}\text { Q1 3.3 (SE 0.07) V. } \\
\text { Q5 3.2 (SE 0.06) }\end{array}$ & $P=0.10$ \\
\hline & & & & & $\mathrm{HbA1c}(\%)$ & & & & $\begin{array}{l}\text { Q1 } 5.50 \text { (SE } 0.04) v \text {. } \\
\text { Q5 5.49 (SE 0.04) }\end{array}$ & $P=0.83$ \\
\hline & & & & & Insulin (pmol/l) & & & & Q1 91.7 (SE 4.9) $v$. & $P=0.13$ \\
\hline & & & & & C-peptide (ng/ml) & & & & $\begin{array}{r}\text { Q1 } 2.13 \text { (SE 0.08) V. } \\
\text { Q5 1.84 (SE 0.08) }\end{array}$ & $P=0.03$ \\
\hline & & & & & Leptin (ng/ml) & & & & $\begin{array}{r}\text { Q1 } 9.02(\text { SE 1.03) V. } \\
\text { Q5 8.03 (SE 1.04) }\end{array}$ & $P=0.03$ \\
\hline & & & & & CRP (mg/l) & & & & $\begin{array}{r}\text { Q1 } 0.92 \text { (SE 1.07) v. } \\
\text { Q5 } 0.88 \text { (SE 1.07) }\end{array}$ & $P=0.63$ \\
\hline & & & & & Fibrinogen (g/l) & & & & Q1 2.2 (SE 0.03$) \mathrm{V}$. & $P=0.77$ \\
\hline & & & & & IL-6 (pg/ml) & & & & Q1 1.20 (SE 1.04) v & $P=0.87$ \\
\hline & & & & & $\begin{array}{l}\text { Homocysteine } \\
(\mu \mathrm{mol} / \mathrm{l})\end{array}$ & & & & $\begin{array}{r}\text { Q1 } 13.2(\text { SE } 0.6) \mathrm{V} \\
\text { Q5 } 10.9 \text { (SE 0.3) }\end{array}$ & $P<0.001$ \\
\hline Liu, 1999(23) & PCS & NHS I & $75521 \mathrm{~F}$ & USA & $\mathrm{CHD}$ & $\begin{array}{l}2 \cdot 70 \\
\quad \text { serves } / d^{*}\end{array}$ & $\begin{array}{l}0.13 \\
\quad \text { serves/d }\end{array}$ & $\begin{array}{l}\text { Age, BMI, smoking status, } \\
\text { alcohol intake, family history } \\
\text { of MI before } 60 \text { years, self- } \\
\text { reported history of } \\
\text { hypertension or } \\
\text { hypercholesterolaemia, } \\
\text { menopausal status, protein } \\
\text { intake, aspirin use, use of } \\
\text { multiple vitamin or vitamin E } \\
\text { supplements, vigorous } \\
\text { activity, total energy intake } \\
\text { and saturated fat, MUFA, } \\
\text { PUFA and trans fat intake } \\
\text { Whole grain constituent intake: } \\
\text { dietary fibre, folate, vitamin }\end{array}$ & $\begin{array}{c}\text { RR } 0.79(95 \% \mathrm{Cl} \\
0.62,1.01)\end{array}$ & $P=0.01$ \\
\hline Juan, $2017^{(24)}$ & PCS & NHS I and HPFS & $71750 F+42823 M$ & USA & Stroke & $\mathrm{F}: 33.23 \mathrm{~g} / \mathrm{d}$ & $\mathrm{F}: 4.43 \mathrm{~g} / \mathrm{d}$ & $\begin{array}{l}\text { Age, BMI, smoking status, } \\
\text { alcohol intake, physical } \\
\text { activity, family history of } \\
\text { diabetes mellitus, cancer } \\
\text { and heart disease, } \\
\text { multivitamin use, } \\
\text { hypertension or high } \\
\text { cholesterol at baseline, total } \\
\text { energy intake, modified } \\
\text { alternative eating index } \\
\text { score (did not include whole } \\
\text { grains), menopausal status } \\
\text { and postmenopausal } \\
\text { hormone use }\end{array}$ & $\begin{array}{c}\mathrm{HR} 1.03(95 \% \mathrm{Cl} \\
0.88,1.21)\end{array}$ & $P=0.35$ \\
\hline
\end{tabular}




\section{N British Journal of Nutrition}

Table 5. Continued

\begin{tabular}{|c|c|c|c|c|c|c|c|c|c|c|}
\hline $\begin{array}{l}\text { Study first author, } \\
\text { year (reference) }\end{array}$ & Study & Cohort & Participants & Country & Outcome & $\begin{array}{l}\text { Highest } \\
\text { intake }\end{array}$ & $\begin{array}{l}\text { Lowest } \\
\text { intake }\end{array}$ & Covariates & Results & $P$ \\
\hline & & & & & & M: $47.90 \mathrm{~g} / \mathrm{d}$ & M: $5.92 \mathrm{~g} / \mathrm{d}$ & & $\begin{array}{c}\text { HR } 1.06(95 \% \mathrm{Cl} \\
0.85,1.32)\end{array}$ & $P=0.37$ \\
\hline & & & & & & N/A & N/A & & $\begin{array}{c}\text { Pooled HR } 1.04 \\
(95 \% \text { Cl } 0.91 \\
1.19)\end{array}$ & $P=0.97$ \\
\hline \multirow[t]{2}{*}{ Fung, 2002 ${ }^{(25)}$} & PCS & HPFS & $42898 \mathrm{M}$ & USA & T2DM & 3.2 serves/d & 0.4 serves $/ d$ & $\begin{array}{l}\text { Age, time period, physical } \\
\text { activity, energy intake, } \\
\text { missing FFQ, smoking } \\
\text { status, family history of } \\
\text { diabetes, alcohol intake, fruit } \\
\text { intake, vegetable intake and } \\
\text { BMI }\end{array}$ & $\begin{array}{l}\text { RR } 0.70(95 \% \mathrm{Cl} \\
0.57,0.85)\end{array}$ & $P=0.0006$ \\
\hline & & & & & & & & Cereal fibre intake & $\begin{array}{c}\text { RR } 0.98(95 \% \mathrm{Cl} \\
0.76,1.26)\end{array}$ & $P=0.98$ \\
\hline \multirow[t]{2}{*}{ Montonen, $2003^{(26)}$} & PCS & FMC & $4316 \mathrm{~F}+\mathrm{M}$ & Finland & T2DM & $302 \mathrm{~g} / \mathrm{d}$ & $79 \mathrm{~g} / \mathrm{d}$ & $\begin{array}{l}\text { Age, sex, geographic area, } \\
\text { energy intake, smoking } \\
\text { status, BMl and intake of } \\
\text { fruit, berries and vegetables }\end{array}$ & $\begin{array}{c}\mathrm{RR} 0.65(95 \% \mathrm{Cl} \\
0.36,1.18)\end{array}$ & $P=0.02$ \\
\hline & & & & & & & & Cereal fibre intake & $\begin{array}{c}\text { RR } 1.14(95 \% \mathrm{Cl} \\
0.49,2.66)\end{array}$ & $P=0.63$ \\
\hline \multirow[t]{2}{*}{ Meyer, $2000^{(27)}$} & PCS & IWHS & $\begin{array}{l}35988 \\
\text { postmenopausal } F\end{array}$ & USA & T2DM & $\begin{array}{l}20.5 \text { serves/ } \\
\text { week }\end{array}$ & $\begin{array}{l}1.0 \text { serves/ } \\
\text { week }\end{array}$ & $\begin{array}{l}\text { Age, total energy intake, BMI, } \\
\text { waist:hip ratio, education, } \\
\text { smoking status, alcohol } \\
\text { intake and physical activity }\end{array}$ & $\begin{array}{c}\text { RR } 0.79(95 \% \mathrm{Cl} \\
0.65,0.96)\end{array}$ & $P=0.0089$ \\
\hline & & & & & & & & Cereal fibre intake & $\begin{array}{c}\mathrm{RR} 0.93(95 \% \mathrm{Cl} \\
0.75,1.16)\end{array}$ & $P=0.46$ \\
\hline \multirow[t]{3}{*}{ McKeown, 2004 ${ }^{(28)}$} & Cross-sectional & FOS & $2834 M+F$ & USA & Mean HOMA-IR & $\begin{array}{l}20.4 \text { serves/ } \\
\text { week }\end{array}$ & $\begin{array}{l}0.9 \text { serves/ } \\
\text { week }\end{array}$ & $\begin{array}{l}\text { Age, sex, BMI, waist:hip ratio, } \\
\text { smoking status, total energy } \\
\text { intake, alcohol intake, } \\
\text { percentage saturated fat, } \\
\text { percentage PUFA, } \\
\text { multivitamin use, physical } \\
\text { activity and treatment for BP }\end{array}$ & $\begin{array}{r}\text { Q1 6.8 }(6 \cdot 6,7.1) v . \\
\text { Q5 6.6 (6.4, 6.9) }\end{array}$ & $P=0.05$ \\
\hline & & & & & $\begin{array}{l}\text { Metabolic } \\
\text { syndrome }\end{array}$ & & & $\begin{array}{l}\text { Cereal fibre intake } \\
\text { Sex, age, smoking status, total } \\
\text { energy intake, alcohol } \\
\text { intake, percentage saturated } \\
\text { fat, percentage PUFA, } \\
\text { multivitamin use and } \\
\text { physical activity }\end{array}$ & $\begin{array}{l}\text { Q1 } 6.8 v . \text { Q5 } 6.7 \\
\text { OR } 0.67(95 \% \mathrm{Cl} \\
0.48,0.91)\end{array}$ & $\begin{array}{l}P=0.34 \\
P=0.01\end{array}$ \\
\hline & & & & & & & & Cereal fibre intake & $\begin{array}{c}\text { OR } 0.77(95 \% \mathrm{Cl} \\
0.55,1.09)\end{array}$ & $P=0.20$ \\
\hline \multirow[t]{4}{*}{ Huang, 2015 (29) } & PCS & $\begin{array}{l}\text { NIH-AARP Diet } \\
\text { and Health } \\
\text { Study }\end{array}$ & $367442 \mathrm{M}+\mathrm{F}$ & USA & CVD death & $1.20 \mathrm{oz} / \mathrm{d}$ & $0.13 \mathrm{oz} / \mathrm{d}$ & $\begin{array}{l}\text { Age, sex, smoking status, race/ } \\
\text { ethnicity group, alcohol } \\
\text { intake, education level, } \\
\text { marital status, health status, } \\
\text { obesity, physical activity, } \\
\text { intake of red meat, fruit and } \\
\text { vegetable, total energy } \\
\text { intake and hormone usage }\end{array}$ & $\begin{array}{l}\mathrm{HR} 0.83(95 \% \mathrm{Cl} \\
0.78,0.88)\end{array}$ & $P<0.001$ \\
\hline & & & & & & & & Cereal fibre intake & $\begin{array}{c}\mathrm{HR} 0.95(95 \% \mathrm{Cl} \\
0.88,1.03)\end{array}$ & $P=0.19$ \\
\hline & & & & & All-cause death & & & $\begin{array}{l}\text { Age, sex, smoking status, race/ } \\
\text { ethnicity group, alcohol } \\
\text { intake, education level, } \\
\text { marital status, health status, } \\
\text { obesity, physical activity, } \\
\text { intake of red meat, fruit and } \\
\text { vegetable, total energy } \\
\text { intake and hormone usage }\end{array}$ & $\begin{array}{l}\text { HR } 0.83(95 \% \mathrm{Cl} \\
0.81,0.86)\end{array}$ & $P<0.0001$ \\
\hline & & & & & & & & Cereal fibre intake & $\begin{array}{l}\text { HR } 0.94(95 \% \mathrm{Cl} \\
0.90,0.97)\end{array}$ & $P=0.002$ \\
\hline
\end{tabular}


Table 5. Continued

\begin{tabular}{|c|c|c|c|c|c|c|c|c|c|c|}
\hline $\begin{array}{l}\text { Study first author, } \\
\text { year (reference) }\end{array}$ & Study & Cohort & Participants & Country & Outcome & $\begin{array}{l}\text { Highest } \\
\text { intake }\end{array}$ & $\begin{array}{l}\text { Lowest } \\
\text { intake }\end{array}$ & Covariates & Results & $P$ \\
\hline \multirow[t]{3}{*}{ Jacobs, $2000^{(30)}$} & PCS & IWHS & $\begin{array}{l}11040 \\
\text { postmenopausal F }\end{array}$ & USA & $\mathrm{CHD}$ death & $\begin{array}{l}4.7 \mathrm{~g} \text { whole } \\
\text { grain fibre/ } \\
8368 \mathrm{~kJ} \\
(2000 \\
\mathrm{kcal})\end{array}$ & $\begin{array}{l}1.3 \mathrm{~g} \text { whole } \\
\text { grain fibre/ } \\
8368 \mathrm{~kJ} \\
(2000 \\
\text { kcal) }\end{array}$ & $\begin{array}{l}\text { Age, energy intake, } \\
\text { educational status, marital } \\
\text { status, hypertension, } \\
\text { diabetes, heart disease, } \\
\text { cancer, BMl, waist:hip ratio, } \\
\text { age at first birth, physical } \\
\text { activity score, smoking } \\
\text { status, pack years of } \\
\text { cigarette smoking, alcohol } \\
\text { intake, vitamin supplement } \\
\text { use, hormone replacement } \\
\text { therapy use, percentage } \\
\text { energy from total fat, } \\
\text { saturated fat and } \\
\text { carbohydrates, intake per } \\
4184 \mathrm{~kJ}(1000 \mathrm{kcal}) \text { of fibre } \\
\text { from non-grain sources, intake } \\
\text { of red meat, fish and seafood }\end{array}$ & $\begin{array}{l}\text { HR } 0.89(95 \% \mathrm{Cl} \\
0.66,1.20)\end{array}$ & $P>0.05$ \\
\hline & & & & & Other CVD death & & & & $\begin{array}{c}\mathrm{HR} 0.75(95 \% \mathrm{Cl} \\
0.54,1.06)\end{array}$ & $P>0.05$ \\
\hline & & & & & All-cause death & & & & $\begin{array}{c}\text { HR } 0.83(95 \% \mathrm{Cl} \\
0.73,0.94)\end{array}$ & $P<0.05$ \\
\hline \multirow[t]{2}{*}{ Erkkila, $2005^{(31)}$} & PCS & ERA & $\begin{array}{l}229 \text { postmenopausal F } \\
\text { with coronary } \\
\text { atherosclerosis }\end{array}$ & USA & $\begin{array}{l}\text { Change in min. } \\
\text { coronary artery } \\
\text { diameter }(\mathrm{mm})\end{array}$ & $\begin{array}{l}>6 \text { serves/ } \\
\text { week }\end{array}$ & $\begin{array}{l}\leq 6 \text { serves/ } \\
\text { week }\end{array}$ & $\begin{array}{l}\text { Age, location of coronary } \\
\text { segment, BMl, smoking } \\
\text { status, use of cholesterol- } \\
\text { lowering medication and } \\
\text { hormone replacement } \\
\text { therapy, diabetes, education, } \\
\text { clinic, time of follow-up, } \\
\text { alcohol intake, coronary } \\
\text { artery bypass, percutaneous } \\
\text { transluminal coronary } \\
\text { angioplasty, energy intake, } \\
\text { saturated fat, PUFA and } \\
\text { cholesterol intake, serum } \\
\text { total, HDL- and LDL- } \\
\text { cholesterol, TAG and } \\
\text { systolic BP }\end{array}$ & $\begin{array}{l}\text { Lowest intake: } \\
-0.10 \text { (sD 0.02) v. } \\
\text { highest intake: } \\
-0.06 \text { (sD 0.02) }\end{array}$ & $P=0.04$ \\
\hline & & & & & $\begin{array}{l}\text { Change stenosis } \\
(\%)\end{array}$ & & & & $\begin{array}{l}\text { Lowest intake: } 3.62 \\
\text { (sD } 0.86) v . \\
\text { highest intake: } \\
1.69 \text { (SD } 0.90 \text { ) }\end{array}$ & $P=0.09$ \\
\hline \multirow[t]{3}{*}{ Newby, $2007^{(32)}$} & Cross-sectional & BLSA & $1516 F+M$ & USA & $\begin{array}{l}\text { Diastolic BP } \\
(\mathrm{mmHg})\end{array}$ & $45.4 \mathrm{~g} / \mathrm{d}$ & $0.62 \mathrm{~g} / \mathrm{d}$ & $\begin{array}{l}\text { Age, age }{ }^{2} \text {, sex, total energy } \\
\text { intake, decade of visit, race, } \\
\text { education, vitamin } \\
\text { supplement use, smoking } \\
\text { status, percentage energy } \\
\text { from saturated fat, alcohol } \\
\text { and refined grains, BMl, use } \\
\text { of BP medication and } \\
\text { hypertension }\end{array}$ & $\begin{array}{r}\text { Q1 } 79.8 \text { (SE } 0.6) v \\
\text { Q5 } 79.2(\text { SE } 0.7)\end{array}$ & $P=0.42$ \\
\hline & & & & & $\begin{array}{c}\text { Systolic BP } \\
\text { (mmHg) }\end{array}$ & & & & $\begin{array}{l}\text { Q1 } 129.2(\text { SE 1.0) } v . \\
\text { Q5 128.3 (SE 1.0) }\end{array}$ & $P=0.79$ \\
\hline & & & & & $\begin{array}{l}\text { Total cholesterol } \\
(\mathrm{mmol} / \mathrm{l})\end{array}$ & $45.6 \mathrm{~g} / \mathrm{d}$ & $0.63 \mathrm{~g} / \mathrm{d}$ & $\begin{array}{l}\text { Age, age }{ }^{2} \text {, sex, total energy } \\
\text { intake, decade of visit, race, } \\
\text { education, vitamin } \\
\text { supplement use, smoking } \\
\text { status, percentage energy } \\
\text { from saturated fat, alcohol } \\
\text { and refined grains, BMl, use } \\
\text { of lipid-lowering medication } \\
\text { and hypercholesterolaemia }\end{array}$ & $\begin{array}{r}\text { Q1 } 5.71 \text { (SE 0.06) v. } \\
\text { Q5 5.49 (SE 0.06) }\end{array}$ & $P=0.02$ \\
\hline
\end{tabular}




\begin{tabular}{|c|c|c|c|c|c|c|c|c|c|c|}
\hline $\begin{array}{l}\text { Study first author, } \\
\text { year (reference) }\end{array}$ & Study & Cohort & Participants & Country & Outcome & $\begin{array}{l}\text { Highest } \\
\text { intake }\end{array}$ & $\begin{array}{l}\text { Lowest } \\
\text { intake }\end{array}$ & Covariates & Results & $P$ \\
\hline & & & & & $\begin{array}{l}\text { HDL-cholesterol } \\
(\mathrm{mmol} / \mathrm{l})\end{array}$ & $54.0 \mathrm{~g} / \mathrm{d}$ & $4.1 \mathrm{~g} / \mathrm{d}$ & & $\begin{array}{l}\text { Q1 } 1.27 \text { (SE 0.02) } v \text {. } \\
\text { Q5 1.22 (SE 0.02) }\end{array}$ & $P=0.07$ \\
\hline & & & & & $\begin{array}{l}\text { LDL-cholesterol } \\
(\mathrm{mmol} / \mathrm{l}) \\
\text { TAG (mmol/l) }\end{array}$ & $54.8 \mathrm{~g} / \mathrm{d}$ & $3.9 \mathrm{~g} / \mathrm{d}$ & & $\begin{array}{r}\text { Q1 } 3.16(\mathrm{SE} 0.06) v . \\
\text { Q5 } 2.96 \text { (SE 0.06) } \\
\text { Q1 1.23 (SE 0.05) v. }\end{array}$ & $\begin{array}{l}P=0.04 \\
P=0.22\end{array}$ \\
\hline & & & & & $\begin{array}{l}\text { Fasting glucose } \\
(\mathrm{mmol} / \mathrm{l})\end{array}$ & $45.4 \mathrm{~g} / \mathrm{d}$ & $0.56 \mathrm{~g} / \mathrm{d}$ & $\begin{array}{l}\text { Age, age }{ }^{2} \text {, sex, total energy } \\
\text { intake, decade of visit, race, } \\
\text { education, vitamin } \\
\text { supplement use, smoking } \\
\text { status, percentage energy } \\
\text { from saturated fat, alcohol } \\
\text { and refined grains, BMl, oral } \\
\text { hypoglycaemic medication } \\
\text { and diabetes }\end{array}$ & $\begin{array}{r}\text { Q5 1.16 (SE 0.05) } \\
\text { Q1 5.49(SE 0.06) v. } \\
\text { Q5 5.49 (SE 0.06) }\end{array}$ & $P=0.57$ \\
\hline & & & & & $\begin{array}{c}2 \mathrm{~h} \text { glucose } \\
(\mathrm{mmol} / \mathrm{l})\end{array}$ & $50.6 \mathrm{~g} / \mathrm{d}$ & $1.1 \mathrm{~g} / \mathrm{d}$ & & $\begin{array}{r}\text { Q1 } 8.24 \text { (SE 0.17) } v . \\
\text { Q5 7.32 (SE 0.17) }\end{array}$ & $P=0.006$ \\
\hline & & & & & $\begin{array}{l}\text { Fasting insulin } \\
(\mathrm{pmol} / \mathrm{l})\end{array}$ & $51.5 \mathrm{~g} / \mathrm{d}$ & $2 \cdot 2 \mathrm{~g} / \mathrm{d}$ & & $\begin{array}{r}\text { Q1 71.6 (SE 3.9) } v \text {. } \\
\text { Q5 71.8 (SE 4.1) }\end{array}$ & $P=0.90$ \\
\hline & & & & & $2 \mathrm{~h}$ insulin (pmol/l) & $51.7 \mathrm{~g} / \mathrm{d}$ & $2.4 \mathrm{~g} / \mathrm{d}$ & & $\begin{array}{r}\text { Q1 } 479 \text { (SE 38.0) v. } \\
\text { Q5 } 414(\mathrm{SE} 39.1)\end{array}$ & $P=0.43$ \\
\hline & & & & & BMI $\left(\mathrm{kg} / \mathrm{m}^{2}\right)$ & $46.0 \mathrm{~g} / \mathrm{d}$ & $0.65 \mathrm{~g} / \mathrm{d}$ & $\begin{array}{l}\text { Age, age }^{2}, \text { sex, total energy } \\
\text { intake, decade of visit, race, } \\
\text { education, vitamin } \\
\text { supplement use, smoking } \\
\text { status, percentage energy } \\
\text { from saturated fat, alcohol } \\
\text { and refined grains }\end{array}$ & $\begin{array}{l}\text { Q1 } 25.5 \text { (SE 0.2) v. } \\
\text { Q5 24.8 (SE 0.2) }\end{array}$ & $P<0.0001$ \\
\hline & & & & & Weight (kg) & & & & $\begin{array}{r}\text { Q1 } 75.0 \text { (SE 0.7) } v \\
\text { Q5 } 72.6 \text { (SE 0.2) }\end{array}$ & $P=0.004$ \\
\hline & & & & & $\begin{array}{l}\text { Waist } \\
\text { circumference } \\
(\mathrm{cm})\end{array}$ & $49.3 \mathrm{~g} / \mathrm{d}$ & $0.94 \mathrm{~g} / \mathrm{d}$ & & $\begin{array}{r}\text { Q1 87.4 (SE 0.6) V. } \\
\text { Q5 85.0 (SE 0.6) }\end{array}$ & $P=0.002$ \\
\hline \multirow[t]{3}{*}{ McKeown, $2009^{(33)}$} & Cross-sectional & $\begin{array}{r}3 \text { year trial (vitamin } \\
\mathrm{K} \text { and bone loss) }\end{array}$ & $324 M+F$ & USA & $\operatorname{BMl}\left(\mathrm{kg} / \mathrm{m}^{2}\right)$ & $\begin{array}{l}2 \cdot 86 \\
\text { serves/d }\end{array}$ & $\begin{array}{l}0.21 \\
\quad \text { serves } / \mathrm{d}\end{array}$ & $\begin{array}{l}\text { Age, sex, total energy intake, } \\
\text { percentage energy from fat, } \\
\text { physical activity, smoking } \\
\text { status, alcohol intake and } \\
\text { multivitamin use }\end{array}$ & $\begin{array}{l}\mathrm{Q} 126 \cdot 8(25 \cdot 7 \\
28 \cdot 1) v . \mathrm{Q} 425 \cdot 8 \\
(24 \cdot 6,27 \cdot 1)\end{array}$ & $P=0.08$ \\
\hline & & & & & Body fat (\%) & & & & $\begin{array}{l}\text { Q1 } 34.5(32 \cdot 7 \\
36 \cdot 3) v . \mathrm{Q} 432 \cdot 1 \\
(30 \cdot 1,34 \cdot 1)\end{array}$ & $P=0.02$ \\
\hline & & & & & Trunk fat mass (\%) & & & & $\begin{array}{c}\text { Q1 } 43.0(40.4 \\
45.5) \text { v. Q4 } 39.4 \\
(36.7,42 \cdot 1)\end{array}$ & $P=0.02$ \\
\hline \multirow[t]{5}{*}{$\begin{array}{l}\text { Van de Viiver, } \\
2007^{(34)}\end{array}$} & Cross-sectional & NLCS & $4237 \mathrm{M}+\mathrm{F}$ & Netherlands & BMI $\left(\mathrm{kg} / \mathrm{m}^{2}\right)$ & $\mathrm{N} / \mathrm{A}$ & $\mathrm{N} / \mathrm{A}$ & $\begin{array}{l}\text { Age, energy intake, intake of } \\
\text { animal protein, education, } \\
\text { smoking status, number of } \\
\text { cigarettes, intake of fruit and } \\
\text { vegetables }\end{array}$ & $\begin{array}{l}\text { M: } \beta-0.03(-0.05 \\
-0.02) \\
\mathrm{F}: \beta-0.04(-0.06 \\
-0.01)\end{array}$ & $\begin{array}{l}P<0.01 \\
P<0.01\end{array}$ \\
\hline & & & & & Overweight risk & & & & $\begin{array}{l}\text { M: OR } 0.98(95 \% \\
\text { Cl } 0.96,0.99)\end{array}$ & $P<0.01$ \\
\hline & & & & & & & & & $\begin{array}{l}\text { F: OR } 0.98(95 \% \\
\quad \text { Cl } 0.96,0.99)\end{array}$ & $P<0.01$ \\
\hline & & & & & Obesity risk & & & & $\begin{array}{l}\text { M: OR } 0.90(95 \% \\
\mathrm{Cl} 0.84,0.98)\end{array}$ & $P<0.05$ \\
\hline & & & & & & & & & $\begin{array}{l}\mathrm{F}: \text { OR } 0.96(95 \% \\
\quad \mathrm{Cl} 0.93,0.99)\end{array}$ & $P<0.05$ \\
\hline
\end{tabular}

HPFS, Health Professionals Follow-up Study; NHS, Nurses' Health Study; M, male; F, female; CRP, C-reactive protein; PCS, prospective cohort study; MI, myocardial infarction; RR, relative risk; HR, hazard ratio; T2DM, type 2 diabetes mellitus; FMC, Finnish Mobile Clinic Health Examination Survey; IWHS, lowa Women's Health Study; FOS, Framingham Offspring Study; HOMA-IR, homoeostatic model assessment of insulin resistance; ERA, Estrogen Replacement and Atherosclerosis trial; BLSA, Baltimore Longitudinal Study of Aging; BP, blood pressure; NLCS, Netherlands Cohort Study; N/A, not available.

* One whole grain serve (s): one slice bread; one cup breakfast cereal, rice, pasta, other grain, popcorn, cooked oatmeal; one tablespoon bran, wheat germ. 


\section{N British Journal of Nutrition}

Table 6. Characteristics of studies within expanded analysis exploring associations of cereal fibre intake with outcomes related to CVD

\begin{tabular}{|c|c|c|c|c|c|c|c|c|c|c|}
\hline $\begin{array}{l}\text { Study first author, } \\
\text { year (reference) }\end{array}$ & Study & Cohort & Participants & Cou-ntry & Outcome & Highest intake & Lowest intake & Covariates & Results & $P$ \\
\hline Montonen, $2003^{(26)}$ & PCS & FMC & $4316 \mathrm{~F}+\mathrm{M}$ & Finland & T2DM & $24.5-111 \mathrm{~g} / \mathrm{d}$ & $0.47-12.0 \mathrm{~g} / \mathrm{d}$ & $\begin{array}{l}\text { Age, sex, geographic area, } \\
\text { energy intake, smoking } \\
\text { status, BMl and intake of } \\
\text { fruit, berries and vegetables }\end{array}$ & $\begin{array}{c}\text { RR } 0.39(95 \% \mathrm{Cl} \\
0.20,0.77)\end{array}$ & $P=0.01$ \\
\hline \multirow[t]{2}{*}{ Meyer, $2000^{(27)}$} & PCS & IWHS & $35988 \mathrm{~F}$ & USA & T2DM & $9.43 \mathrm{~g} / \mathrm{d}$ & $2.66 \mathrm{~g} / \mathrm{d}$ & $\begin{array}{l}\text { Age, total energy intake, BMI, } \\
\text { waist:hip ratio, education, } \\
\text { smoking status, alcohol } \\
\text { intake and physical activity }\end{array}$ & $\begin{array}{c}\mathrm{RR} 0.64(95 \% \mathrm{Cl} \\
0.53,0.79)\end{array}$ & $P=0.0001$ \\
\hline & & & & & & & & Whole grain intake & $\begin{array}{c}\text { RR } 0.66(95 \% \mathrm{Cl} \\
0.53,0.83)\end{array}$ & $P=0.0001$ \\
\hline \multirow[t]{3}{*}{ McKeown, 2004 } & Cross-sectional & FOS & $2834 M+F$ & USA & Mean HOMA-IR & $8.0 \mathrm{~g} / \mathrm{d}$ & $2.6 \mathrm{~g} / \mathrm{d}$ & $\begin{array}{l}\text { Age, sex, BMI, waist:hip ratio, } \\
\text { smoking status, total energy } \\
\text { intake, alcohol intake, } \\
\text { percentage saturated fat, } \\
\text { percentage PUFA, } \\
\text { multivitamin use, physical } \\
\text { activity and treatment for } \\
\text { blood pressure }\end{array}$ & $\begin{array}{r}\text { Q1 } 6.8(6.5,7 \cdot 0) v \\
\text { Q5 } 6 \cdot 6(6 \cdot 3,6 \cdot 8)\end{array}$ & $P=0.02$ \\
\hline & & & & & & & & Whole grain intake & Q1 6.9 v. Q5 6.5 & $P=0.003$ \\
\hline & & & & & $\begin{array}{l}\text { Metabolic } \\
\text { syndrome }\end{array}$ & & & $\begin{array}{l}\text { Sex, age, smoking status, total } \\
\text { energy intake, alcohol } \\
\text { intake, percentage saturated } \\
\text { fat, percentage PUFA, } \\
\text { multivitamin use and } \\
\text { physical activity }\end{array}$ & $\begin{array}{c}\text { OR } 0.62(95 \% \mathrm{Cl} \\
0.45,0.86)\end{array}$ & $P=0.002$ \\
\hline \multirow[t]{2}{*}{ Huang, $2015^{(29)}$} & PCS & $\begin{array}{l}\text { NIH-AARP } \\
\text { Diet and } \\
\text { Health } \\
\text { Study }\end{array}$ & $367442 \mathrm{M}+\mathrm{F}$ & USA & CVD death & $10.22 \mathrm{~g} / \mathrm{d}$ & $2.02 \mathrm{~g} / \mathrm{d}$ & $\begin{array}{l}\text { Age, sex, smoking status, race/ } \\
\text { ethnicity group, alcohol } \\
\text { intake, education level, } \\
\text { marital status, health status, } \\
\text { obesity, physical activity, } \\
\text { intake of red meat, fruit and } \\
\text { vegetable, total energy } \\
\text { intake and hormone usage }\end{array}$ & $\begin{array}{c}\mathrm{HR} 0.80(95 \% \mathrm{Cl} \\
0.75,0.85)\end{array}$ & $P<0.0001$ \\
\hline & & & & & All-cause death & & & & $\begin{array}{c}\text { HR } 0.81(95 \% \mathrm{Cl} \\
0.790 .84)\end{array}$ & $P<0.0001$ \\
\hline \multirow[t]{2}{*}{ Erkkila, $2005^{(31)}$} & PCS & ERA & $\begin{array}{l}229 \text { postmenopausal } \\
\mathrm{F} \text { with coronary } \\
\text { atherosclerosis }\end{array}$ & USA & $\begin{array}{l}\text { Change in min. } \\
\text { coronary artery } \\
\text { diameter (mm) }\end{array}$ & $\begin{array}{r}>3 \mathrm{~g} / 4184 \mathrm{~kJ} \\
(1000 \mathrm{kcal})\end{array}$ & $\begin{array}{r}\leq 3 \mathrm{~g} / 4184 \mathrm{~kJ} \\
(1000 \mathrm{kcal})\end{array}$ & $\begin{array}{l}\text { Age, location of coronary } \\
\text { segment, BMI, smoking } \\
\text { status, use of cholesterol- } \\
\text { lowering medication and } \\
\text { hormone replacement } \\
\text { therapy, diabetes, education, } \\
\text { clinic, time of follow-up, } \\
\text { alcohol intake, coronary } \\
\text { artery bypass, percutaneous } \\
\text { transluminal coronary } \\
\text { angioplasty, energy intake, } \\
\text { saturated fat, PUFA and } \\
\text { cholesterol intake, serum } \\
\text { total, HDL-, and LDL- } \\
\text { cholesterol, TAG and } \\
\text { systolic blood pressure }\end{array}$ & $\begin{array}{c}\text { Lowest: }-0.09 \mathrm{v} . \\
\text { highest: }-0.04\end{array}$ & $P=0.03$ \\
\hline & & & & & $\begin{array}{l}\text { Change stenosis } \\
(\%)\end{array}$ & & & & $\begin{array}{r}\text { Lowest: } 3.44 \mathrm{v} \text {. } \\
\text { highest: } 1.79\end{array}$ & $P=0.10$ \\
\hline
\end{tabular}




\section{Nes British Journal of Nutrition}

\begin{tabular}{|c|c|c|c|c|c|c|c|c|c|c|}
\hline $\begin{array}{l}\text { Study first author, } \\
\text { year (reference) }\end{array}$ & Study & Cohort & Participants & Cou-ntry & Outcome & Highest intake & Lowest intake & Covariates & Results & $P$ \\
\hline \multirow[t]{13}{*}{ Newby, 2007(32) } & Cross-sectional & BLSA & $1516 \mathrm{~F}+\mathrm{M}$ & USA & $\begin{array}{l}\text { Diastolic BP } \\
(\mathrm{mmHg})\end{array}$ & $9.43 \mathrm{~g} / \mathrm{d}$ & $2.19 \mathrm{~g} / \mathrm{d}$ & $\begin{array}{l}\text { Age, age }{ }^{2} \text {, sex, total energy } \\
\text { intake, decade of visit, race, } \\
\text { education, vitamin } \\
\text { supplement use, smoking } \\
\text { status, percentage energy } \\
\text { from saturated fat and } \\
\text { alcohol, BMl, use of blood } \\
\text { pressure-lowering } \\
\text { medication and } \\
\text { hypertension }\end{array}$ & $\begin{array}{r}\text { Q1 } 79.5 \text { (SE } 0.8) v \\
\text { Q5 } 79.2(\mathrm{SE} 0.8)\end{array}$ & $P=0.90$ \\
\hline & & & & & $\begin{array}{c}\text { Systolic BP } \\
(\mathrm{mmHg})\end{array}$ & & & & $\begin{array}{r}\text { Q1 } 128.7 \text { (SE 1.0) } v \\
\text { Q5 129.7 (SE 1.0) }\end{array}$ & $P=0.27$ \\
\hline & & & & & $\begin{array}{l}\text { Total cholesterol } \\
(\mathrm{mmol} / \mathrm{l})\end{array}$ & $9.56 \mathrm{~g} / \mathrm{d}$ & $2.2 \mathrm{~g} / \mathrm{d}$ & $\begin{array}{l}\text { Age, age }^{2}, \text { sex, total energy } \\
\text { intake, decade of visit, race, } \\
\text { education, vitamin } \\
\text { supplement use, smoking } \\
\text { status, percentage energy } \\
\text { from saturated fat and } \\
\text { alcohol, BMI, use of lipid- } \\
\text { lowering medication and } \\
\text { hypercholesterolaemia }\end{array}$ & $\begin{array}{r}\text { Q1 } 5.73(\text { SE } 0.06) v \\
\text { Q5 } 5.44(\text { SE } 0.06)\end{array}$ & $P=0.005$ \\
\hline & & & & & $\begin{array}{l}\text { HDL-cholesterol } \\
(\mathrm{mmol} / \mathrm{l})\end{array}$ & $11.49 \mathrm{~g} / \mathrm{d}$ & $2.9 \mathrm{~g} / \mathrm{d}$ & & \multirow{4}{*}{ 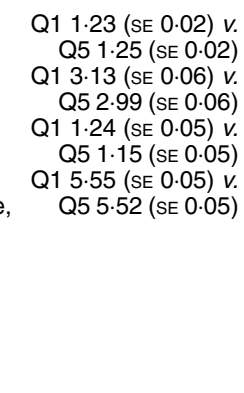 } & \\
\hline & & & & & $\begin{array}{l}\text { LDL-cholesterol } \\
(\mathrm{mmol} / \mathrm{l})\end{array}$ & & & & & $P=0.07$ \\
\hline & & & & & $\mathrm{TAG}(\mathrm{mmo} / \mathrm{l})$ & $9.58 \mathrm{~g} / \mathrm{d}$ & $2.2 \mathrm{~g} / \mathrm{d}$ & & & $P=0.12$ \\
\hline & & & & & $\begin{array}{l}\text { Fasting glucose } \\
(\mathrm{mmol} / \mathrm{l})\end{array}$ & $9.67 \mathrm{~g} / \mathrm{d}$ & $2.27 \mathrm{~g} / \mathrm{d}$ & $\begin{array}{l}\text { Age, age }{ }^{2}, \text { sex, total energy } \\
\text { intake, decade of visit, race, } \\
\text { education, vitamin } \\
\text { supplement use, smoking } \\
\text { status, percentage energy } \\
\text { from saturated fat and } \\
\text { alcohol, BMI, oral } \\
\text { hypoglycaemic medication } \\
\text { and diabetes }\end{array}$ & & $P=0.95$ \\
\hline & & & & & $\begin{array}{c}2 \mathrm{~h} \text { glucose } \\
(\mathrm{mmol} / \mathrm{l})\end{array}$ & $10.42 \mathrm{~g} / \mathrm{d}$ & $2.39 \mathrm{~g} / \mathrm{d}$ & & \multirow{3}{*}{$\begin{array}{r}\text { Q1 } 8.05 \text { (SE 0.21) } v \text {. } \\
\text { Q5 6.48 (SE 0.21) } \\
\text { Q1 68.9 (SE 4.0) v. } \\
\text { Q5 73.0 (SE 4.0) } \\
\text { Q1 } 438(\text { SE 38.8) v. } \\
\text { Q5 } 413(\text { SE 38.2) }\end{array}$} & $P=0.02$ \\
\hline & & & & & $\begin{array}{l}\text { Fasting insulin } \\
(\mathrm{pmol} / \mathrm{l})\end{array}$ & $11.63 \mathrm{~g} / \mathrm{d}$ & $2.54 \mathrm{~g} / \mathrm{d}$ & & & $P=0.68$ \\
\hline & & & & & $2 \mathrm{~h}$ insulin $(\mathrm{pmol} / \mathrm{l})$ & & & & & $P=0.33$ \\
\hline & & & & & BMI $\left(\mathrm{kg} / \mathrm{m}^{2}\right)$ & $9.51 \mathrm{~g} / \mathrm{d}$ & $2.19 \mathrm{~g} / \mathrm{d}$ & \multirow[t]{3}{*}{$\begin{array}{l}\text { Age, age', sex, total energy } \\
\text { intake, decade of visit, race, } \\
\text { education, vitamin } \\
\text { supplement use, smoking } \\
\text { status, percentage energy } \\
\text { from saturated fat, alcohol } \\
\text { and refined grains }\end{array}$} & $\begin{array}{r}\mathrm{Q} 125.7 \text { (SE } 0.2) v \\
\text { Q5 24.3 (SE } 0.2)\end{array}$ & $P<0.0001$ \\
\hline & & & & & Weight $(\mathrm{kg})$ & & & & \multirow{2}{*}{$\begin{array}{r}\text { Q1 } 75.6 \text { (SE 0.7) } v . \\
\text { Q5 71.4 (SE 0.8) } \\
\text { Q1 87.4 (SE 0.6) v. } \\
\text { Q5 84.1 (SE 0.6) }\end{array}$} & $P=0.0004$ \\
\hline & & & & & $\begin{array}{l}\text { Waist } \\
\text { circumference } \\
(\mathrm{cm})\end{array}$ & $9.74 \mathrm{~g} / \mathrm{d}$ & $2.31 \mathrm{~g} / \mathrm{d}$ & & & $P<0.0001$ \\
\hline
\end{tabular}




\section{N British Journal of Nutrition}

Table 6. Continued

\begin{tabular}{|c|c|c|c|c|c|c|c|c|c|c|}
\hline $\begin{array}{l}\text { Study first author, } \\
\text { year (reference) }\end{array}$ & Study & Cohort & Participants & Cou-ntry & Outcome & Highest intake & Lowest intake & Covariates & Results & $P$ \\
\hline \multirow[t]{3}{*}{ McKeown $2009^{(33)}$} & \multirow[t]{3}{*}{ Cross-sectional } & \multirow[t]{3}{*}{$\begin{array}{l}3 \text { year trial } \\
\quad \text { (vitamin } \mathrm{K} \\
\text { and bone } \\
\text { loss) }\end{array}$} & \multirow[t]{3}{*}{$324 M+F$} & \multirow[t]{3}{*}{ USA } & BMI $\left(\mathrm{kg} / \mathrm{m}^{2}\right)$ & \multirow[t]{3}{*}{$9 \cdot 3 \mathrm{~g} / \mathrm{d}$} & \multirow[t]{3}{*}{$2.4 \mathrm{~g} / \mathrm{d}$} & \multirow[t]{3}{*}{$\begin{array}{l}\text { Age, sex, total energy intake, } \\
\text { percentage energy from fat, } \\
\text { physical activity, smoking } \\
\text { status, alcohol intake and } \\
\text { multivitamin use }\end{array}$} & $\begin{array}{l}\text { Q1 } 27.3(26 \cdot 1 \\
28 \cdot 6) \text { v. Q4 } 25 \cdot 4 \\
(24 \cdot 2,26 \cdot 7)\end{array}$ & $P=0.01$ \\
\hline & & & & & Body fat (\%) & & & & $\begin{array}{c}\text { Q1 34.7 (32.8 } \\
\text { 36.6) v. Q4 } 31.5 \\
(29.4,33.5)\end{array}$ & $P=0.004$ \\
\hline & & & & & Trunk fat mass (\%) & & & & $\begin{array}{c}\text { Q1 } 42.8(40.2, \\
45.4) v . \mathrm{Q} 437.8 \\
(35.0,40.6)\end{array}$ & $P=0.001$ \\
\hline \multirow[t]{5}{*}{$\begin{array}{l}\text { Van de Vijver } \\
2007^{(34)}\end{array}$} & \multirow[t]{5}{*}{ Cross-sectional } & \multirow[t]{5}{*}{ NLCS } & \multirow[t]{5}{*}{$4237 \mathrm{M}+\mathrm{F}$} & \multirow[t]{5}{*}{ Nether-lands } & BMI $\left(\mathrm{kg} / \mathrm{m}^{2}\right)$ & \multirow[t]{5}{*}{$\mathrm{N} / \mathrm{A}$} & \multirow[t]{5}{*}{ N/A } & \multirow[t]{5}{*}{$\begin{array}{l}\text { Age, energy intake, intake of } \\
\text { animal protein, education, } \\
\text { smoking status, number of } \\
\text { cigarettes, intake of fruits } \\
\text { and vegetables }\end{array}$} & 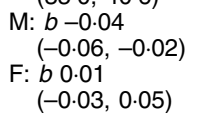 & $\begin{array}{l}P<0.01 \\
P>0.05\end{array}$ \\
\hline & & & & & Overweight risk & & & & $\begin{array}{l}\text { M: OR } 0.98(95 \% \\
\quad \text { Cl } 0.96,0.99)\end{array}$ & $P<0.01$ \\
\hline & & & & & & & & & $\begin{array}{l}\mathrm{F}: \text { OR } 1.00(95 \% \\
\mathrm{Cl} 0.97,1.02)\end{array}$ & $P>0.05$ \\
\hline & & & & & Obesity risk & & & & $\begin{array}{l}\text { M: OR } 0.97(95 \% \\
\text { Cl } 0.92,1.02)\end{array}$ & $P>0.05$ \\
\hline & & & & & & & & & $\begin{array}{l}\text { F: OR } 1.03(95 \% \\
\text { Cl } 0.98,1.07)\end{array}$ & $P>0.05$ \\
\hline
\end{tabular}

PCS, prospective cohort study; FMC, Finnish Mobile Clinic Health Examination Survey; F, female; M, male; T2DM, type 2 diabetes mellitus; RR, relative risk; IWHS, lowa Women's Health Study; FOS, Framingham Offspring Study; HOMAIR, homoeostatic model assessment of insulin resistance; HR, hazard ratio; ERA, Estrogen Replacement and Atherosclerosis trial; BLSA, Baltimore Longitudinal Study of Aging; BP, blood pressure; NLCS, Netherlands Cohort Study; N A, not available. 


\section{Nes British Journal of Nutrition}

Table 7. Characteristics of studies within expanded analysis exploring associations of bran intake with outcomes related to CVD

\section{Study first author, year}

(reference)

Study

Cohort

Participants

Country Outcome

Bran

Highest intake

sectional NHS

$468 \mathrm{M}+470 \mathrm{~F}$ USA

HbA1c (\%)

Total $^{\star} \quad 13.56 \mathrm{~g} / \mathrm{d}$

$1.42 \mathrm{~g} / \mathrm{d}$

Covariates

Age, sex, smoking status,

BMI, physical activity,

Q1 5.45 (SE 0.04) v. $P=0.53$

hypercholesterolaemia,

intake, intake of fruits,

vegetables, germ, sucrose

and fructose

Q5 5.50 (SE 0.05)

Insulin (pmol/l)

C-peptide (ng/ml)

Leptin $(\mathrm{ng} / \mathrm{ml})$

Total cholesterol

$(\mathrm{mmol} / \mathrm{l})$

HDL-cholesterol

(mmol/l)

LDL-cholesterol

$(\mathrm{mmol} / \mathrm{l})$

CRP (mg/l)

Fibrinogen $(g / l)$

IL-6 (pg/ml)

Homocysteine

( $\mu \mathrm{mol} / \mathrm{l})$

Liu, 1999(23)

PCS

NHS

$75521 \mathrm{~F}$

USA

Added† 1.0 serves/dł 0 serves/d

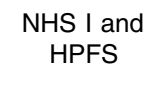
71750
$\mathrm{F}+42823$
$\mathrm{M}$

USA Stroke

186.8 (SE 4.9) v. $P=0.14$ Q5 76.4 (SE 3.5)

Q1 2.11 (SE 0.10) v. $P=0.05$

Q5 1.84 (SE 0.06)

Q1 8.67 (SE 1.04) v. $P=0.88$

Q5 8.5 (SE 1.04)

Age, sex, smoking status, BMI, physical activity,

hypercholesterolaemia,

alcohol intake, total energy

intake, intake of fruits,

vegetables, germ

saturated fat, MUFA and

PUFA

15.8 (SE 0.09) $v$

Q5 5.6 (SE 0.07) 
T2DM among healthy participants within the NHS I (relative risk (RR) $0.75,95 \%$ CI $0.68,0.83, P<0.001$ and RR $0.72,95 \% \mathrm{CI}$ $0 \cdot 65,0 \cdot 80, P<0 \cdot 001$, respectively) and the NHS II (RR 0.86, $95 \%$ CI $0.72,1.02, P=0.03$ and RR $0.84,95 \%$ CI $0.71,1.00$, respectively) ${ }^{(19)}$; however, the inverse association with total bran intake within the NHS II only reached borderline significance $(P=0.07)$

Whole grain intake was associated with a $19 \%$ lower risk of hypertension $^{(20)}$ (RR 0.81, 95\% CI 0.75, 0.87, P<0.0001) and a $16 \%$ lower risk of $\mathrm{CHD}^{(5)}$ (HR 0.84, $95 \% \mathrm{CI} 0 \cdot 71,0 \cdot 98, P=0 \cdot 02$ ) in the HPFS, when comparing the highest quartile of intake to lowest. However, after adjusting for cereal fibre intake ${ }^{(20)}$ and whole grain constituents including dietary fibre ${ }^{(5)}$, both of these association were attenuated $(P=0.23$ and $P=0.06$, respectively), suggesting some significance of the cereal fibre component towards the whole grain association. Bran intake was also inversely associated with risk of both hypertension and CHD (RR 0.85, 95\% CI 0.78, 0.92, $P=0.002$ and HR 0.72, 95\% CI $0 \cdot 61,0 \cdot 84, P<0 \cdot 001$, respectively), and the association with lower risk of $\mathrm{CHD}$ remained significant after adjusting for constituents $(P<0 \cdot 001)$.

Whole grain intake compared with bran and cereal fibre intake. Within the HPFS, whole grain, bran and cereal fibre intake were associated with better weight management over time ${ }^{(21)}$. The association was stronger for whole grain intake than for cereal fibre or bran intake. For every $40 \mathrm{~g} / \mathrm{d}$ increase in whole grain intake, 8 -year weight gain was $1.1 \mathrm{~kg}$ lower $(P<0.0001)$, while for every $20 \mathrm{~g} / \mathrm{d}$ increase in cereal fibre or bran intake, 8 -year weight gain was $0.81 \mathrm{~kg}(P=0.0002)$ and $0.36 \mathrm{~kg}$ lower $(P=0.01)$, respectively, indicating a slightly stronger association for whole grain intake.

Lower risks of CVD mortality and all-cause mortality were found for high whole grain, cereal fibre and bran (total and added) intake in a cohort of 7822 women with existing $\mathrm{T} 2 \mathrm{DM}^{(4)}$. However, after adjustment for lifestyle and dietary factors, only the associations for total and added bran intake remained significant for both outcomes. A $64 \%$ lower risk of CVD mortality (RR 0.36, $95 \%$ CI $0.24,0.54, P<0.001$ ) and a $55 \%$ lower risk of all-cause mortality (RR $0.45,95 \%$ CI $0 \cdot 36$, $0.57, P<0.001)$ were observed for those consuming the highest intake of added bran compared with non-consumers.

Whole grain, bran and cereal fibre were significantly inversely related to C-reactive protein (CRP) and TNF receptor 2 levels in a fair quality analysis of women with T2DM within the NHS I ${ }^{(17)}$, but no association was found with E-selectin or intercellular adhesion molecule 1 levels. Notably, within this study no adjustment was made for other dietary factors that may be correlated with whole grain intake and influence inflammation, such as PUFA intake.

\section{Expanded analysis}

Eleven of thirteen articles within the expanded analysis found a significant inverse association between whole grain intake and at least one CVD-related outcome analysed ${ }^{(22,23,25-29,31-34)}$, though six of these found the association no longer significant 
Table 8. Classification of study design as per National Health and Medical Research Council (NHMRC) level of evidence guidelines and classification of quality as per National Institutes of Health $(\mathrm{NIH})$ quality assessment tool for observational cohort and cross-sectional studies

\begin{tabular}{|c|c|c|}
\hline Reference & $\begin{array}{l}\text { NHMRC level } \\
\text { of evidence }\end{array}$ & $\begin{array}{c}\mathrm{NIH} \text { quality assessment } \\
\text { tool rating }\end{array}$ \\
\hline \multicolumn{3}{|l|}{ Initial analysis } \\
\hline $\mathrm{He}(2010)^{(4)}$ & $\|$ & Good \\
\hline Jensen $(2004)^{(5)}$ & II & Good \\
\hline Qi $(2006)^{(17)}$ & IV & Fair \\
\hline Wu $(2015)^{(18)}$ & II & Good \\
\hline de Munter $(2007)^{(19)}$ & II & Good \\
\hline Flint $(2009)^{(20)}$ & III-2 & Good \\
\hline Koh Banerjee $(2004)^{(21)}$ & II & Good \\
\hline \multicolumn{3}{|l|}{ Expanded analysis } \\
\hline Jensen $(2006)^{(22)}$ & IV & Fair \\
\hline Liu $(1999)^{(23)}$ & II & Good \\
\hline Juan $(2017)^{(24)}$ & II & Good \\
\hline Fung (2002) (25) & II & Good \\
\hline Montonen (2003) ${ }^{(26)}$ & II & Fair \\
\hline Meyer $(2000)^{(27)}$ & II & Fair \\
\hline McKeown (2004) & IV & Fair \\
\hline Huang $(2015)^{(29)}$ & II & Good \\
\hline Jacobs $(2000)^{(30)}$ & II & Fair \\
\hline Erkkila $(2005)^{(31)}$ & III-2 & Fair \\
\hline Newby $(2007)^{(32)}$ & IV & Fair \\
\hline McKeown (2009) ${ }^{(33)}$ & IV & Fair \\
\hline Van de Vijver (2009) ${ }^{(34)}$ & IV & Fair \\
\hline
\end{tabular}

after adjustment for cereal fibre intake ${ }^{(23,25-29)}$, indicating cereal fibre as a key component in the association. All eight articles that analysed cereal fibre intake separately found a significant inverse association with at least one CVD-related outcome ${ }^{(26-29,31-34)}$. One article investigating bran intake found mixed association ${ }^{(22)}$, while the other two found significant

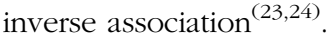

Whole grain intake compared with bran intake. One fair quality analysis ${ }^{(22)}$ considered association of whole grain and bran intake with lipid profile, markers of glycaemic control and inflammation. The highest consumers of whole grains (quintile 5; Q5) had 2.9\% lower total cholesterol $(P=0 \cdot 02), 5 \cdot 3 \%$ lower HDL-cholesterol $(P=0.05)$ and $2.1 \%$ lower LDL-cholesterol than the lowest consumers (quintile 1; Q1), although the association with LDL-cholesterol did not reach significance $(P=0 \cdot 10)$. Highest whole grain intake $(Q 5)$ was also associated with $17 \cdot 4 \%$ lower levels of homocysteine as well as lower concentrations of C-peptide $(-13.6 \%, P=0.03)$ and leptin ($11.0 \%, P=0.03)$. Other markers examined, including HbA1c, were not associated with whole grain intake. Similar associations were seen when examining bran intake separately but were only borderline statistically significant, with the exception of homocysteine concentrations $(-10.9 \%, P=0.02)$. Leptin was not associated with bran intake $(-2 \cdot 0 \%, P=0 \cdot 88)$.

Whole grain and bran intake were both associated with lower incidence of CHD in a good quality prospective cohort study ${ }^{(23)}$ (RR $0.75,95 \%$ CI $0.59,0.95, P=0.01$ and RR $0.63,95 \%$ CI 0.42 , $0 \cdot 95, P=0 \cdot 001$, respectively), with participants consuming one serving of added bran per d observing a $37 \%$ lower risk of $\mathrm{CHD}$ compared with non-consumers. These significant associations were found after adjustment for several related lifestyle and diet covariates including smoking status, physical activity and fatty acid composition of diet, however, the association with whole grain intake was attenuated after further adjustment for grain constituents including dietary fibre $(P=0.07)$. High total bran intake was associated with $11 \%$ lower risk of stroke (HR 0.89 , $95 \%$ CI $0.79,1.0, P=0.004)$ in a separate good quality pooled cohort study, whereas whole grain intake showed no association $^{(24)}$.

Whole grain intake compared with cereal fibre intake. Whole grain intake was associated with a $21 \%$ (95\% CI 0.65 , $0 \cdot 96, P=0.0089)$ to $35 \%(95 \% \mathrm{CI} 0 \cdot 36,1 \cdot 18, P=0 \cdot 02)$ lower risk of T2DM comparing the highest intake to the lowest intake in three studies (one good quality, two fair quality) ${ }^{(25-27)}$. Importantly, the two fair quality studies failed to adjust for potentially important covariates including physical activity ${ }^{(26)}$ and related dietary factors such as fruit and vegetable intake ${ }^{(27)}$. A fourth study of fair quality found a $33 \%$ lower risk (95\% CI 0.48, 0.91, $P=0.01)$ of the metabolic syndrome ${ }^{(28)}$. The association completely disappeared when adjusting for cereal fibre in all four cases. The three fair quality studies also observed association for cereal fibre intake separately and found that the highest intake was associated with a $36 \%$ (95\% CI $0.53,0.79$, $P=0.0001)$ to $61 \%(95 \%$ CI $0 \cdot 20,0 \cdot 77, P=0 \cdot 01)$ lower risk of T2DM, and a $38 \%(95 \%$ CI $0 \cdot 45,0 \cdot 86, P=0.002)$ lower risk of the metabolic syndrome. Meyer et al. ${ }^{(27)}$ adjusted this result for whole grain intake, and the lower risk was only slightly reduced to $34 \%(95 \%$ CI $0.53,0.83, P=0.0001)$.

The association of whole grain and cereal fibre intake with all-cause mortality and CVD-mortality was explored in a large, good quality analysis of 367442 US participants ${ }^{(29)}$. High whole grain intake was associated with a $17 \%$ lower risk of both outcomes (95\% CI 0.81, 0.86, $P<0.0001$ and 95\% CI 0.78, 0.88, $P<0.001$, respectively). Once adjusted for cereal fibre, the association with all-cause mortality was attenuated to 6\% (95\% CI $0.90,0.97, P=0.002$ ), and the association with CVD mortality completely disappeared (HR 0.95, $95 \%$ CI 0.88, 1·03, $P=0 \cdot 188$ ). High cereal fibre intake, analysed separately within the same study, was associated with very similar lower risk of all-cause and CVD mortality. In a smaller, fair quality study ${ }^{(30)}$, whole grain consumers tended to have lower risk of all-cause mortality (HR 0.83, 95\% CI 0.73, 0.94, $P<0.05$ ) compared with refined grain consumers.

The association of whole grain and cereal fibre intake to progression of coronary atherosclerosis among 229 women were analysed within a fair quality study ${ }^{(31)}$. Women consuming above the median intake of whole grains ( $>6$ serves/week) and cereal fibre intake $(>3 \mathrm{~g} / 4184 \mathrm{~kJ}(1000 \mathrm{kcal}))$ had a significantly smaller change in minimum coronary artery diameter over the 3-year period compared with those consuming under the median $(-0.06 v .-0.10 \mathrm{~mm}, P=0.04$ and $-0.04 v .-0.09 \mathrm{~mm}$, $P=0.03$, respectively).

Two fair quality cross-sectional articles examined whole grain and cereal fibre intake in relation to glycaemic control. In the Framingham Offspring Study, mean homoeostasis model assessment-estimated insulin resistance (HOMA-IR) was borderline significantly lower in the highest quintile of whole grain 
intake (quintile 5; Q5) compared with the lowest (quintile 1; Q1) (Q1 6.8 v. Q5 6.6, $P=0.05$ ); however, this association disappeared once adjusted for cereal fibre ${ }^{(28)}$. In contrast, the association between high cereal fibre intake and lower mean HOMA-IR remained after adjusting for whole grain intake (Q1 $6.9 v$. Q5 6.5, $P=0.003$ ). Within the Baltimore Longitudinal Study of Aging, higher whole grain and cereal fibre intake were similarly associated with lower postprandial glucose (quintile 1 (Q1) $8.24 \mathrm{mmol} / 1 v$. quintile 5 (Q5) $7.23 \mathrm{mmol} / 1, P=0.006$ and Q1 $8.05 \mathrm{mmol} / \mathrm{l} \quad v$. Q5 $6.48 \mathrm{mmol} / 1, \quad P=0.02$, respectively); however, no association with fasting glucose, fasting insulin or postprandial insulin were found ${ }^{(32)}$

Within the same study, the highest consumers of whole grain and cereal fibre had significantly lower total cholesterol than the lowest consumers (Q1 $5.79 \mathrm{mmol} / \mathrm{l} v$. Q5 $5.49 \mathrm{mmol} / \mathrm{l}, P=0.02$ and Q1 $5.73 \mathrm{mmol} / \mathrm{l} v$. Q5 $5.44 \mathrm{mmol} / \mathrm{l}, P=0.005$, respectively). The highest consumers of both intake also had lower LDLcholesterol than the lowest consumers, though this was borderline significant for cereal fibre intake (Q1 $3.13 \mathrm{mmol} / \mathrm{l} v$. Q5 $2.9 \mathrm{mmol} / \mathrm{l}, P=0.07$ ), and somewhat negatively, the highest consumers of whole grain intake had lower HDL-cholesterol, though not significantly (Q1 $1.27 \mathrm{mmol} / \mathrm{l} v$. Q5 $1.22 \mathrm{mmol} / \mathrm{l}$, $P=0 \cdot 07)$.

Participants consuming the highest intake of whole grain and cereal fibre had significantly lower anthropometric body measurements compared with those consuming the lowest intake in three cross-sectional analyses ${ }^{(32-34)}$, though notably two of the three studies did not perform an adjustment for physical activity within their multivariate models ${ }^{(32,34)}$. Within the Netherlands Cohort Study, it was estimated that every $1 \mathrm{~g} / \mathrm{d}$ higher intake of whole grain was associated with a 0.03 and $0.04 \mathrm{~kg} / \mathrm{m}^{2}$ lower BMI in men and women, respectively $(P<0.01 \text { for both })^{(34)}$. This meant that a decrease of $1 \mathrm{~kg} / \mathrm{m}^{2}$ BMI corresponded to a 33 and $25 \mathrm{~g}$ increase in whole grain intake. Every $1 \mathrm{~g}$ of cereal fibre intake was similarly associated with a $0.04 \mathrm{~kg} / \mathrm{m}^{2}$ lower BMI in men $(P<0.01)$, but no association was found for women. Within the same cohort, a high intake of whole grain was associated with a slightly lower risk of being overweight and obese in both men (OR 0.98, 95\% CI 0.96, 0.99, $P<0 \cdot 01$ and OR $0 \cdot 90,95 \%$ CI $0 \cdot 84,0.98, P<0.05$, respectively) and women (OR $0.98,95 \%$ CI 0.96, 0.99, $P<0.01$ and OR 0.96, $95 \%$ CI 0.93, 0.99, $P<0.05$, respectively), while a high intake of cereal fibre was only associated with a lower risk of being overweight in men (OR 0.98, 95\% CI 0.96, 0.99, P<0.01). van de Vijver et al. also conducted a prospective analysis of a smaller cohort of 1257 participants, with follow-up ranging 1-5 years, but no association was found between baseline intake and anthropometric measures.

\section{Discussion}

Within the review, the association between whole grains, bran and cereal fibre and lower risk of CVD-related outcomes were evident. In the expanded analysis, cereal fibre and whole grain (including added bran) intake were similarly associated with lower risk of CVD mortality, and the fibre component tended to explain the association ${ }^{(29)}$, whereas fibre-rich bran was strongly associated with lower risk of stroke incidence ${ }^{(24)}$. The inverse association between the risk of CVD-related outcomes and cereal fibre intake may be due to favourable effects on factors likely to influence the development of the disease such as serum lipids ${ }^{(35)}$, glucose and insulin metabolism ${ }^{(36)}$, blood pressure $^{(37)}$ and inflammation ${ }^{(38)}$. These factors may also play a role in slowing progression of CVD, as higher intake of cereal fibre and whole grain products were both associated with less progression of coronary atherosclerosis in postmenopausal women with established $\mathrm{CHD}^{(31)}$. The differences in progression $(0.04 \mathrm{~mm}$ less progression in high whole grain consumers; $0.05 \mathrm{~mm}$ less progression in high cereal fibre consumers) were somewhat comparable with treatment effects reported in statin trials $(0 \cdot 06-0.08 \mathrm{~mm})^{(39)}$.

Interestingly when studies assessed added bran intake (bran added to food during processing), some of the strongest inverse association with CVD-related outcomes were found. As added bran, as opposed to total bran, does not include bran intake consumed as part of whole grain foods, there is less chance of it being simply a surrogate marker for whole grain intake, providing a more precise insight into the benefits of bran alone. In the study by He et al. ${ }^{(4)}$ of women with T2DM, only added bran was significantly associated with lower CVD-related and allcause mortality after adjustment for related lifestyle and dietary factors. While this study was underpowered to detect modest associations; and therefore, the non-significant associations found for whole grain and cereal fibre may be due to limited power, the same strong inverse association with CVD mortality and all-cause-mortality was found for added bran intake in healthy participants ${ }^{(18)}$. Furthermore, added bran had the strongest inverse association with $\mathrm{CHD}^{(5,23)}$. One possible explanation for the consistently strong inverse associations found for added bran is that within these populations, bran is sourced mainly from only two sources, wheat bran and oat bran $^{(5)}$. Oat bran is one of the highest sources of the soluble fibre $\beta$-glucan, which is consistently associated with reduced serum total LDL-cholesterol in clinical trials ${ }^{(40,41)}$.

However, the direct association within the cross-sectional studies of whole grain, cereal fibre and total bran intake to serum cholesterol levels were less consistent ${ }^{(22,32)}$. Again, association may have been stronger if specifically analysing intake of high-soluble fibre grains. Cross-sectional studies are inherently unable to capture the impact of long-term trends in intake, and sample sizes were relatively small within both studies. For example, while a $4.7 \%$ lower LDL-cholesterol level, as found in one study comparing highest consumers of cereal fibre with lowest consumers ${ }^{(32)}$, may appear clinically relevant to reduced CVD risk ${ }^{(42)}$, it did not quite reach statistical significance $(P=0 \cdot 07)$.

It is thought that whole grains may contribute to weight loss through the effect of fibre on satiation and satiety ${ }^{(43)}$, leading to lower energy intake. In addition, the secretion of particular gut hormones such as cholecystokinin may contribute to satiety or alter glucose homoeostasis ${ }^{(44)}$, and soluble fibre may improve insulin sensitivity by slowing the absorption of macronutrients, indirectly affecting body weight ${ }^{(43)}$. While the inverse association within the HPFS between whole grain, added bran and cereal fibre intake and weight gain over an 8 -year period were 
statistically significant, the weight change differences between the highest and lowest intake were modest ${ }^{(21)}$. Considering the findings for whole grains and fibre intake effect on weight regulation in clinical trials tend to be inconsistent ${ }^{(45-47)}$, further research investigating this relationship is needed.

Within this review, whole grain intake tended to be associated with lower incidence of T2DM and the metabolic syndrome, but this association consistently disappeared after adjustments for cereal fibre intake. The one study that did not perform adjustments for cereal fibre intake found similar risk reductions between whole grain and $\operatorname{bran}^{(19)}$. High-soluble fibre foods have been found to reduce post-prandial glucose and insulin response ${ }^{(48)}$ and high-fibre diets may improve insulin sensitivity and lower insulin secretion ${ }^{(49)}$. Interestingly, two studies within the review found that insoluble fibre but not soluble fibre was associated with lower risk of $\mathrm{T}_{2} \mathrm{DM}^{(26,27)}$. Insoluble fibre may also slow the absorption of foods ${ }^{(50)}$, but it is also suggested that a quicker transit time, achieved with highinsoluble fibre intake, allows less time for carbohydrates to be absorbed in the upper jejunum, thus relieving insulin demand ${ }^{(51)}$. Both whole grain and cereal fibre intake were associated with significantly lower HOMA-IR, a measure of insulin resistance, in the Framingham cohort even after adjustment for relevant lifestyle and dietary factors. However, the whole grain association disappeared after adjustment for cereal fibre ${ }^{(28)}$.

In addition to the fibre content, micronutrients within whole grains such as Mg may also play a role in insulin sensitivity and reduced risk of T2DM. In a small study of elderly participants, $4.5 \mathrm{~g}$ of $\mathrm{Mg}$ daily for 4 weeks improved glucose and insulin responses ${ }^{(52)}$. Within our review, the importance of $\mathrm{Mg}$ intake was inconclusive. Some studies found that $\mathrm{Mg}$ intake could not explain the whole grain association ${ }^{(19,26)}$, while another found $\mathrm{Mg}$ was independently associated with reduced risk of T2DM, even after adjustment for cereal fibre ${ }^{(27)}$. Importantly, this study did not adjust for any other related dietary factors that may confound these associations. Overall, the findings suggest that the fibre component of whole grains and bran may play the major role in the inverse association with T2DM. Other nutrients such as Mg may also contribute.

CRP was the only inflammatory marker regularly assessed. Elevated CRP concentrations, a marker of inflammation, have been found to independently predict CVD in the general population $^{(53)}$ and people with $\mathrm{T}_{2} \mathrm{DM}^{(54)}$, and the American Heart Association has concluded that the risk of CVD is lower when the CRP level is below $1 \mathrm{mg} / \mathrm{l}^{(55)}$. Here, high whole grain, bran and cereal fibre intake were significantly associated with lower CRP concentrations in the same cohort of women with $\mathrm{T}_{2} \mathrm{DM}^{(17)}$. Within a healthy population, high whole grain and bran intake tended to be associated with lower CRP concentrations; however, after adjustment for dietary and lifestyle factors, the association completely disappeared for whole grain and became non-significant for bran ${ }^{(22)}$. Hyperglycaemia may, in part, influence production of pro-inflammatory cytokines ${ }^{(56,57)}$, so whole grain and cereal fibre intake may assist in inhibiting inflammation through an effect on blood glucose control.

The findings of this review suggest that fibre may play a significant role in the cardio-protective association of whole grains. Whole grain, bran and cereal fibre intake were associated with similarly lowered risks of most outcomes assessed, and when tested, cereal fibre often accounted considerably for whole grain associations, particularly for T2DM. When bran was separated from whole grains as in the initial analysis, intake of bran, particularly added bran, had equal or stronger inverse association with various CVD-related outcomes to that of whole grains. As bran contains the majority of cereal fibre within grains, this may further support the notion that fibre contributes considerably to the protective associations of grains. Of course, it is important to note that bran cannot be interpreted simply as a direct measure of cereal fibre, due to the abundance of minerals, phytochemicals and phyto-oestrogens contained within the aleurone layer ${ }^{(58)}$. In the HPFS cohort, adjusting for fibre and micronutrient constituents attenuated the inverse association between whole grains and CHD but did not have an appreciable effect on the strong inverse association for added bran $^{(5)}$. Further, Jacobs et $a l{ }^{(30)}$ found that consumers of fibre from whole grain sources (which included bran) compared with fibre from refined grain sources tended to have lower risk of allcause mortality but not specifically CVD mortality. We cannot disregard the possibility that these bioactive compounds within the bran aleurone layer, botanically linked to fibre, may contribute to the inverse associations found ${ }^{(30)}$.

Another important consideration in examining associations between whole grains and CVD-related outcomes is the diet and lifestyle of whole grain consumers, beyond whole grain consumption. Whole grain intake may be associated with an overall healthier lifestyle and better diet quality ${ }^{(59)}$, and some of these associated factors such as higher physical activity levels, lower incidence of smoking and higher fruit and vegetable intake may confound or contribute to any associations attributed to whole grain intake. Within the initial analysis, all seven of the good quality articles included careful adjustment for multiple lifestyle and dietary factors in their multivariate models. However, the fair quality article ${ }^{(17)}$ observing associations with markers of inflammation included lifestyle factors within the multivariate model, without inclusion of potentially relevant dietary factors such as polyunsaturated fat intake ${ }^{(60)}$. Similarly, several of the articles included within the expanded analysis failed to adjust for factors such as physical activity status $^{(26,31,32,34)}$, alcohol intake ${ }^{(26)}$ and other dietary factors ${ }^{(27)}$. In some cases, articles reporting on the same study cohorts adjusted for considerably different factors within their multivariate models. As causation cannot be determined from observational studies, it is crucial that careful adjustment is made for all potentially confounding factors. The inconsistency among several articles within the review, particularly within the expanded analysis, may limit the quality of the overall evidence.

Many of the articles within this review also went on to adjust for constituents within the whole grain, including cereal fibre. It was interesting that associations found within the initial analysis were less prone to attenuation after adjustment for cereal fibre than in the expanded analysis. Using an accepted definition of whole grain (including all fractions in natural proportions), and methodical calculation of whole grain, bran and cereal fibre intake, results are less likely to be confounded by imprecise categorisation. We can therefore be more confident that the 
abundance of nutrients expected to be found within whole grains and the separate bran component were accurately captured in estimated intake. Beyond the incorrect inclusion of added bran, it is possible that the presence of non-whole grain (and therefore potentially nutrient poor) grain intake incorrectly captured as whole grain intake within the expanded analysis diluted the overall quality of intake and therefore blunted the contribution of any whole grain nutrients beyond cereal fibre. The noticeable difference in results between initial and expanded analysis suggests that while it is evident that cereal fibre is an important component of whole grain and bran, we cannot disregard contribution from nutrients beyond this.

The inconsistency in definitions and categorisation systems used, and therefore limited amount of evidence based on the correct definition of whole grains, is a considerable limitation of this review. Only three major study cohorts (the NHS I and II and the HPFS) were analysed using the correct definition of whole grains. In addition, while the wide variety of CVD-related outcomes assessed shed light on the various ways whole grain intake may be protective, we were limited in our ability to draw sound conclusions about any one risk factor or outcome, as most outcomes were assessed in few studies, in diverse populations, or using different definitions of whole grain, making a more in-depth study of results by way of a meta-analysis not possible.

\section{Conclusion}

Intake of whole grain, cereal fibre and bran (total and added) appeared to be similarly associated with lower risk of various CVD-related risk factors and outcomes within this review, suggesting that the fibre component of whole grains, located primarily within the bran fraction, may play an important role in the cardio-protective associations observed with whole grain intake. Notably, however, when the accepted definition and precise categorisation of whole grain and bran intake were used, association found were stronger and less prone to attenuation after adjustment for cereal fibre, potentially indicating a role of whole grain nutrients beyond fibre.

The findings from this review are interesting in consideration of the promotion of grain foods in public health initiatives and dietetic practice. Potentially, an overly restricted focus on whole grain cereal foods may deter consumption of high-fibre nonwhole grain foods such as bran-based cereals. The similar lower risks observed for added bran separate to whole grain across both analyses raise the question of whether current initiatives and health policies should include mention of whole grain and/ or bran foods. Indeed, this question may also be valuable for the food industry, whereby the reformulation of cereal products containing extrinsically sourced cereal fibre or fine bran may overcome sensory barriers to whole grain foods - while still providing cardio-protective benefits. Future adequately powered studies, including intervention studies comparing the effects of whole grain intake with fibre-matched bran or addedcereal fibre intake on CVD-related risk factors and outcomes, are needed to establish the mechanisms behind the protective effects of whole grains and their components as well as the dose-response relationships, where there is currently paucity of data and lack of agreement. These studies are needed before findings can be translated into practical application. Most importantly, there is a crucial need for future research to utilise a consistent, methodical and quantified approach when calculating whole grain intake to ensure future evidence in this area is consistent and accurate and reflects the current definition of whole grain.

\section{Acknowledgements}

The authors thank Professor Chris Seal (Newcastle University) for guidance and support in the design of the review.

This research has been conducted with the support of the Australian Government Research Training Program Scholarship. No other funding has been provided.

E. M. B. and E. J. B. designed the research; E. M. B. and E. J. B. conducted research; E. M. B. and M. J. B. analysed data; E. M. B. and E. J. B. wrote the paper; S. R. reviewed synthesis of data results and reviewed manuscript; E. M. B. had primary responsibility for final content.

The authors declare that there are no conflicts of interest.

\section{Supplementary material}

For supplementary material/s referred to in this article, please visit https://doi.org/10.1017/S000711451900031X

\section{References}

1. World Health Organization (2017) Cardiovascular diseases fact sheet. http://www.who.int/mediacentre/factsheets/fs317/ en/ (accessed February 2018).

2. Pearson TA (2002) AHA Guidelines for Primary Prevention of Cardiovascular Disease and Stroke: 2002 Update: Consensus Panel Guide to Comprehensive Risk Reduction for Adult Patients Without Coronary or Other Atherosclerotic Vascular Diseases. Circulation 106, 388-391.

3. Yusuf S, Hawken S, Ounpuu S, et al. (2004) Effect of potentially modifiable risk factors associated with myocardial infarction in 52 countries (the INTERHEART study): casecontrol study. Lancet 364, 937-952.

4. He M, van Dam RM, Rimm E, et al. (2010) Whole-grain, cereal fiber, bran, and germ intake and the risks of all-cause and cardiovascular disease-specific mortality among women with type 2 diabetes mellitus. Circulation 121, 2162-2168.

5. Jensen MK, Koh-Banerjee MF, Hu FB, et al. (2004) Intakes of whole grains, bran, and germ and the risk of coronary heart disease in men. Am J Clin Nutr 80, 1492-1499.

6. Theuwissen E \& Mensink RP (2008) Water-soluble dietary fibers and cardiovascular disease. Physiol Behav 94, 285-292.

7. Fardet A (2010) New hypotheses for the health-protective mechanisms of whole-grain cereals: what is beyond fibre? Nutr Res Rev 23, 65-134.

8. Cassidy A \& Hooper L (2006) Phytoestrogens and cardiovascular disease. J Br Menopause Soc 12, 49-56.

9. Aune D, Keum N, Giovannucci E, et al. (2016) Whole grain consumption and risk of cardiovascular disease, cancer, and all cause and cause specific mortality: systematic review and doseresponse meta-analysis of prospective studies. BMJ 353, i2716.

10. Ye EQ, Chacko SA, Chou EL, et al. (2012) Greater whole-grain intake is associated with lower risk of type 2 diabetes, cardiovascular disease, and weight gain. J Nutr 142, 1304-1313. 
11. Jacobs DR Jr, Meyer KA, Kushi LH, et al. (1998) Whole-grain intake may reduce the risk of ischemic heart disease death in postmenopausal women: the Iowa Women's Health Study. Am J Clin Nutr 68, 248-257.

12. Ross AB, van der Kamp JW, King R, et al. (2017) Perspective: a definition for whole-grain food products-recommendations from the healthgrain forum. Adv Nutr 8, 525-531.

13. Liberati A, Altman DG, Tetzlaff J, et al. (2009) The PRISMA statement for reporting systematic reviews and meta-analyses of studies that evaluate healthcare interventions: explanation and elaboration. PLoS Med 6, e1000100.

14. American Association of Cereal Chemists International (2017)) Standard definitions and resources: whole grain. http://www. aaccnet.org/initiatives/definitions/Pages/WholeGrain.aspx (accessed September 2017).

15. National Health and Medical Research Council (2000) How to use the evidence: assessment and application of scientific evidence. https://www.nhmrc.gov.au/guidelines-publications/ cp69 (accessed September 2017).

16. National Institute of Health (2014) Quality assessment tool for observational cohort and cross-sectional studies. https://www. nhlbi.nih.gov/health-pro/guidelines/in-develop/cardiovascularrisk-reduction/tools/cohort (accessed September 2017).

17. Qi L, van Dam RM, Liu S, et al. (2006) Whole-grain, bran, and cereal fiber intakes and markers of systemic inflammation in diabetic women. Diabetes Care 29, 207-211.

18. Wu H, Flint AJ, Qi Q, et al. (2015) Association between dietary whole grain intake and risk of mortality: two large prospective studies in US men and women. JAMA Intern Med $\mathbf{1 7 5}$ 373-384.

19. de Munter JSL, Hu FB, Spiegelman D, et al. (2007) Whole grain, bran, and germ intake and risk of type 2 diabetes: a prospective cohort study and systematic review. PloS Med $\mathbf{4}$, e261.

20. Flint AJ, Hu FB, Glynn RJ, et al. (2009) Whole grains and incident hypertension in men. Am J Clin Nutr 90, 493-498.

21. Koh-Banerjee MF, Franz M, Sampson L, et al. (2004) Changes in whole-grain, bran, and cereal fiber consumption in relation to 8-y weight gain among men. Am J Clin Nutr $\mathbf{8 0}$, $1237-1245$.

22. Jensen MK, Koh-Banerjee MF, Sampson L, et al. (2006) Whole grains, bran, and germ in relation to homocysteine and markers of glycemic control, lipids, and inflammation. Am J Clin Nutr 83, 275-283.

23. Liu S, Stampfer MJ, Hu FB, et al. (1999) Whole-grain consumption and risk of coronary heart disease: results from the Nurses' Health Study. Am J Clin Nutr 70, 412-308.

24. Juan J, Liu G, Willett WC, et al. (2017) Whole grain consumption and risk of ischemic stroke: results from 2 prospective cohort studies. Stroke 48, 3203-3209.

25. Fung TT, Hu FB, Pereira MA, et al. (2002) Whole-grain intake and the risk of type 2 diabetes: a prospective study in men. Am J Clin Nutr 76, 535-540.

26. Montonen J, Knekt P, Jarvinen R, et al. (2003) Whole-grain and fiber intake and the incidence of type 2 diabetes. $A m \mathrm{~J}$ Clin Nutr 77, 622-629.

27. Meyer KA, Kushi LH, Jacobs DR, et al. (2000) Carbohydrates, dietary fiber, and incident type 2 diabetes in older women. $A m$ J Clin Nutr 71, 921-930.

28. McKeown NM, Meigs JB, Liu S, et al. (2004) Carbohydrate nutrition, insulin resistance, and the prevalence of the metabolic syndrome in the Framingham Offspring Cohort. Diabetes Care 27, 538-546.

29. Huang T, Xu M, Lee A, et al. (2015) Consumption of whole grains and cereal fiber and total and cause-specific mortality: prospective analysis of 367,442 individuals. BMC Med 13, 59 .
30. Jacobs DR, Pereira MA, Meyer KA, et al. (2000) Fiber from whole grains, but not refined grains, is inversely associated with all-cause mortality in older women: the Iowa Women's Health Study. J Am Coll Nutr 19, Suppl. 3, 326S-330S.

31. Erkkila AT, Herrington DM, Mozaffarian D, et al. (2005) Cereal fiber and whole-grain intake are associated with reduced progression of coronary-artery atherosclerosis in postmenopausal women with coronary artery disease. Am Heart J 150, 94-101.

32. Newby PK, Maras J, Bakun P, et al. (2007) Intake of whole grains, refined grains, and cereal fiber measured with 7-d diet records and associations with risk factors for chronic disease. Am J Clin Nutr 86, 1745-1753.

33. McKeown NM, Yoshida M, Shea MK, et al. (2009) Whole-grain intake and cereal fiber are associated with lower abdominal adiposity in older adults. J Nutr 139, 1950-1955.

34. van de Vijver LP, van den Bosch LM, van den Brandt PA, et al. (2009) Whole-grain consumption, dietary fibre intake and body mass index in the Netherlands cohort study. Eur J Clin Nutr 63, 31-38.

35. Jenkins DJ, Kendall CW, Axelsen M, et al. (2000) Viscous and non-viscous fibres, non-absorbable and low glycaemic index carbohydrates, blood lipids and coronary heart disease. Curr Opin Lipidol 11, 49-56.

36. Juntunen KS, Laaksonen DE, Poutanen KS, et al. (2003) Highfiber rye bread and insulin secretion and sensitivity in healthy postmenopausal women. Am J Clin Nutr 77, 385-391.

37. Appel LJ, Moore TJ, Obarzanek E, et al. (1997) A clinical trial of the effects of dietary patterns on blood pressure. $N$ Engl J Med 336, 1117-1124.

38. King DE, Egan BM \& Geesey ME (2003) Relation of dietary fat and fiber to elevation of C-reactive protein. Am J Cardiol $\mathbf{9 2}$, 1335-1339.

39. Ballantyne CM (1998) Clinical trial endpoints: angiograms, events, and plaque instability. Am J Cardiol 82, Suppl. 1, $5 \mathrm{M}-11 \mathrm{M}$

40. Anderson JW, Gilinsky NH, Deakins DA, et al. (1991) Lipid responses of hypercholesterolemic men to oat-bran and wheat-bran intake. Am J Clin Nutr 54, 678-683.

41. Davy BM, Davy KP, Ho RC, et al. (2002) High-fiber oat cereal compared with wheat cereal consumption favorably alters LDL-cholesterol subclass and particle numbers in middle-aged and older men. Am J Clin Nutr 76, 351-358.

42. National Cholesterol Education Program (NCEP) Expert Panel on Detection, Evaluation, and Treatment of High Blood Cholesterol in Adults (Adult Treatment Panel III) (2002) Third Report of the National Cholesterol Education Program (NCEP) Expert Panel on Detection, Evaluation, and Treatment of High Blood Cholesterol in Adults (Adult Treatment Panel III) final report. Circulation 106, 3143-3421.

43. Koh-Banerjee P \& Rimm EB (2003) Whole grain consumption and weight gain: a review of the epidemiological evidence, potential mechanisms and opportunities for future research. Proc Nutr Soc 62, 25-29.

44. Pereira MA \& Ludwig DS (2001) Dietary fiber and bodyweight regulation. Observations and mechanisms. Pediatr Clin North Am 48, 969-980.

45. Brownlee IA, Moore C, Chatfield M, et al. (2010) Markers of cardiovascular risk are not changed by increased whole-grain intake: the WHOLEheart study, a randomised, controlled dietary intervention. Br J Nutr 104, Suppl. 1, 125S-134S.

46. Rave K, Roggen K, Dellweg S, et al. (2007) Improvement of insulin resistance after diet with a whole-grain based dietary product: results of a randomized, controlled cross-over study in obese subjects with elevated fasting blood glucose. $\mathrm{Br} \mathrm{J}$ Nutr 98, 929-936. 
47. Melanson KJ, Angelopoulos TJ, Nguyen VT, et al. (2006) Consumption of whole-grain cereals during weight loss effects on dietary quality, dietary fiber, magnesium, vitamin B-6, and obesity. J Am Diet Assoc 106, 1380-1388.

48. Jenkins D, Wolever T, Leeds AR, et al. (1978) Dietary fibres, fibre analogues, and glucose tolerance: importance of viscosity. Br Med J 1, 1392-1394.

49. Weickert MO, Mohlig M, Schöfl C, et al. (2006) Cereal fiber improves whole-body insulin sensitivity in overweight and obese women. Diabetes Care 29, 775-780.

50. Anderson JW (1986) Fiber and health: an overview. Am J Gastroenterol 81, 892-897.

51. Cummings JH \& Englyst HN (1987) Fermentation in the human large intestine and the available substrates. Am J Clin Nutr 45, 1243-1255.

52. Paolisso G, Sgambato S, Gambardella A, et al. (1992) Daily magnesium supplements improve glucose handling in elderly subjects. Am J Clin Nutr 55, 1161-1167.

53. Ridker PM, Buring JE, Cook NR, et al. (2003) C-reactive protein, the metabolic syndrome, and risk of incident cardiovascular events: an 8-year follow-up of 14,719 initially healthy American women. Circulation 107, 391-397.

54. Schulze MB, Rimm EB, Li T, et al. (2004) C-reactive protein and incident cardiovascular events among men with diabetes. Diabetes Care 27, 889-894.
55. Pearson TA, Mensah GA, Alexander RW, et al. (2003) Markers of inflammation and cardiovascular disease: application to clinical and public health practice: a statement for healthcare professionals from the Centers for Disease Control and Prevention and the American Heart Association. Circulation 107, 499-511.

56. Gonzalez F, Minium J, Rote NS, et al. (2005) Hyperglycemia alters TNF-alpha release from mononuclear cells in women with polycystic ovary syndrome. J Clin Endocrinol Metab 90 , 5336-5342.

57. Devaraj S, Venugopal SK, Singh U, et al. (2005) Hyperglycemia induces monocytic release of interleukin-6 via induction of protein kinase c-alpha and -beta. Diabetes 54, 85-91.

58. Lillioja S, Neal AL, Tapsell L, et al. (2013) Whole grains, type 2 diabetes, coronary heart disease, and hypertension: links to the aleurone preferred over indigestible fiber. Biofactors 39, 242-258.

59. Kyrø C, Skeie G, Dragsted L, et al. (2011) Intake of whole grains in Scandinavia is associated with healthy lifestyle, socio-economic and dietary factors. Public Health Nutr 14, $1787-1795$.

60. Kris-Etherton PM, Harris WS \& Appel LJ (2003) Omega-3 fatty acids and cardiovascular disease: new recommendations from the American Heart Association. Arterioscler Thromb Vasc Biol 23, 151-152. 